\title{
A RIGOROUS NUMERICAL METHOD FOR THE GLOBAL ANALYSIS OF INFINITE DIMENSIONAL DISCRETE DYNAMICAL SYSTEMS
}

\author{
S. DAY*, O. JUNGE ${ }^{\dagger}$, AND K. MISCHAIKOW $\ddagger$
}

\begin{abstract}
We present a numerical method to prove certain statements about the global dynamics of infinite dimensional maps. The method combines set-oriented numerical tools for the computation of invariant sets and isolating neighborhoods, the Conley index theory and analytic considerations. It not only allows for the detection of a certain dynamical behaviour, but also for a precise computation of the corresponding invariant sets in phase space. As an example computation we show the existence of period points, connecting orbits and chaotic dynamics in the Kot-Schaffer growth-dispersal model for plants.
\end{abstract}

Key words. Conley index, dynamical system, numercial method, infinite dimensional

AMS subject classifications. 37B10, 37B30, 37B50, 37C25, 37C29, 37C70, 37L65, 37M99, 55U99, 65R20

1. Introduction. The techniques described in this paper are motivated by the following three observations:

O1. Most of our knowledge concerning the global dynamics of specific nonlinear systems comes from numerical simulation and as such is lacking in mathematical rigor. In an attempt to rectify this, over the past decade a growing set of techniques has been developed that lead to computer assisted proofs of dynamical structures in low (typically 2 or 3 ) dimensional systems (see $[18,24,1]$ and references therein).

O2. Modelling of phenomena where spatial effects are essential leads to infinite dimensional systems such as partial or functional differential equations, or infinite dimensional maps. However, within these systems the dynamical structures of interest are often low dimensional, e.g. fixed points, periodic orbits, homoclinic and heteroclinic orbits, horseshoes, or low dimensional strange attractors.

O3. In dynamical systems the central objects of interest are invariant sets, i.e. collections of orbits which exist for all time. For a wide variety of infinite dimensional systems, individual solutions which exist globally in time are more regular than the typical functions of the natural phase space (see [9] and references therein).

Keeping these observations in mind our goal is to provide a computationally cheap but accurate numerical method that can be used to prove existence theorems for specific infinite dimensional maps. More precisely, the techniques that we describe are designed for continuous functions $\Phi: X \rightarrow X$ where $X$ is a Hilbert space for which we have an explicit complete orthogonal basis $\left\{\varphi_{k} \mid k=0,1,2, \ldots\right\}$ and the nonlinear terms are polynomial in nature. To provide a concrete demonstration of these ideas we will consider the Kot-Schaffer [15] growth-dispersal model for plants. This consists

\footnotetext{
${ }^{*}$ Center for Dynamical Systems and Nonlinear Studies, Georgia Institute of Technology, Atlanta, Georgia 30322 USA (sday@math.gatech.edu).

${ }^{\dagger}$ Institute of Mathematics, University of Paderborn, 33095 Paderborn, Germany (junge@upb.de)

${ }^{\ddagger}$ Center for Dynamical Systems and Nonlinear Studies, Georgia Institute of Technology, Atlanta, Georgia 30322 USA (mischaik@math.gatech.edu)
} 
of a map $\Phi: L^{2}([-\pi, \pi]) \rightarrow L^{2}([-\pi, \pi])$ of the form

$$
\Phi[a](y):=\frac{1}{2 \pi} \int_{-\pi}^{\pi} b(x, y) g[a](x) d x,
$$

with dispersal kernel $b(x, y)=b(x-y)$ and (polynomial) growth function $g$. In the following example, $g[a](x):=\mu a(x)\left(1-\frac{a(x)}{c(x)}\right)$ with $\mu>0$ and $c \in L^{2}([-\pi, \pi])$. Observe that the regularity of this map is determined by the regularity of the dispersal kernel $b$ and the spatial heterogeneity of $c$ in the nonlinear term.

There are three obvious difficulties that need to be overcome to achieve our goal:

1. Because of the finite nature of a computer it is impossible to compute directly on an infinite dimensional system. Therefore, it is necessary to use an appropriate finite dimensional reduction.

2. Given a finite dimensional system we need to be able to perform two tasks. The first is to locate the different dynamical objects. Since in many cases these objects are dynamically unstable, this is not a trivial task. The second is to rigorously verify that these dynamical structures exist for the finite dimensional system.

3. We need to be able to lift the results of the finite dimensional computations to the full infinite dimensional system.

How these difficulties can be dealt with in a systematic and computationally efficient manner is the subject of this paper and as such is a natural extension of [26]. As the reader might expect, some of the details are fairly technical in nature, and therefore, we take the opportunity of this introduction to provide a broad outline of the procedures which will be developed in the following sections.

Let us begin with a reasonably abstract description of what will be done. We think of $\Phi: X \rightarrow X$ as generating a dynamical system with $a^{\prime}=\Phi(a)$. Recall that $\left\{\varphi_{k} \mid k=0,1,2, \ldots\right\}$ is a complete orthogonal basis for $X$. Let

$$
P_{m}: X \rightarrow X_{m}:=\operatorname{span}\left\{\varphi_{k} \mid k=0,1, \ldots, m-1\right\}
$$

be the orthogonal projection onto the first $m$ modes. The standard Galerkin procedure suggests replacing the study of $\Phi$ by that of the map $f^{(m)}: X_{m} \rightarrow X_{m}$ where $f^{(m)}:=P_{m} \circ \Phi$.

The problem is that if we study the dynamics using $f^{(m)}$ then we do not have any information concerning the errors introduced by the reduction to $X_{m}$ and by the projection $P_{m}$. To get around this problem observe that we can write

$$
\Phi(a)=\Phi\left(P_{m} a\right)+\left(\Phi(a)-\Phi\left(P_{m} a\right)\right) .
$$

In general we cannot hope to determine the right hand term exactly. However if we restrict our attention to a "small" set of $a$, then we may be able to obtain a useful bound on this term. With this in mind, let $W$ be a compact subset of $X_{m}$ and let $V$ be a compact subset of $\left(I-P_{m}\right) X$. Then,

$$
Z:=W \times V
$$

is a compact subset of $X$.

Now assume that it can be shown that for all $a \in Z$

$$
\left\|\Phi(a)-\Phi\left(P_{m} a\right)\right\|<\varepsilon .
$$


Then, for all $a \in Z, \Phi(a)$ lies within an $\varepsilon$-ball of $\Phi\left(P_{m} a\right)$. We want to recast these statements about bounds into the language of dynamical systems. Furthermore, we want this dynamics to be finite dimensional so that we can effectively analyze it. This leads us to consider multivalued or set valued maps $F: W \rightrightarrows \mathbb{R}^{m}$ with the property that for all $a \in Z$,

$$
P_{m} \Phi(a) \in F\left(P_{m} a\right)
$$

Perhaps it is worth noting at this point that if the images of $F$ are "too large" then we will not be able to extract useful information from it. Thus, obtaining good bounds on $\left\|\Phi(a)-\Phi\left(P_{m} a\right)\right\|$ is essential.

At this point we have introduced two functions, the continuous map $f^{(m)}=P_{m}$ 。 $\Phi: W \rightarrow \mathbb{R}^{m}$, which we do not know explicitly, and a multivalued map $F: W \rightrightarrows \mathbb{R}^{m}$ which encloses $f^{(m)}$ in the sense that $f^{(m)}(a) \in F\left(P_{m} a\right)$. It is the function $F$, which implicitly contains the error estimates, that we would like to analyze. However, to directly manipulate an object with the computer it needs to have a combinatorial structure. With this in mind, $W$ is decomposed into a cubical complex on which a combinatorial multivalued map $\mathcal{F}$ that takes grid elements to sets of grid elements is defined. Since each grid element corresponds to a set in $W$, it is easy to pass from the combinatorial map $\mathcal{F}$ to the multivalued map $F$.

This is perhaps a good point to make a remark emphasizing the notational convention adopted for this paper:

- Calligraphed characters represent combinatorial objects or maps;

- Capital letters refer to topological sets or set valued maps;

- Single valued maps (with the exception of $\Phi$ ) are denoted in lower case.

The discussion up to this point has described how one proceeds from the infinite dimensional problem to a combinatorial object that can be analyzed using the computer. The question that remains is how to use this combinatorial information to draw conclusions about the dynamics of $\Phi$. The key tool is the Conley index theory which is a topological generalization of Morse theory. In particular, it can be expressed in terms of homology which is a combinatorial algebraic topological theory. Furthermore, the index can be used to prove the existence of specific dynamical structures such as fixed points, periodic orbits, heteroclinic orbits, and shift dynamics.

As will be detailed later, $\mathcal{F}$ is used to construct isolating neighborhoods and index pairs, and finally to compute the associated Conley index for the map $f^{(m)}$. The important theoretical considerations are that one can pass from $\mathcal{F}$ to a multivalued map $F$ which is an enclosure of $f^{(m)}$ and the Conley index information is preserved through this transition.

The final step is to show that there are conditions under which the Conley index of $f^{(m)}$ is equivalent to the Conley index of $\Phi$ restricted to $Z$. However, since $Z$ is compact, the Conley index theory can be applied immediately to draw conclusions about the existence of dynamic structures for $\Phi$.

At this level of abstraction, it is probably not even clear what are the issues involved in implementing this approach. With this in mind, we shall provide a broad outline of the procedures in the context of the Kot-Schaffer map (1.1).

1.1. Finite Dimensional Reduction. We begin with the reduction to the finite dimensional system, using Fourier modes to decompose $X=L^{2}$. In particular, 
letting $\varphi_{k}(x):=e^{i k x},(1.1)$ becomes equivalent to the countable system of maps

$$
a_{k}^{\prime}=\mu b_{k}\left[a_{k}-\sum_{j+l+n=k} c_{j} a_{l} a_{n}\right], \quad k \in \mathbb{Z}
$$

where $a_{k}, b_{k}, c_{k} \in \mathbb{C}$ are the coefficients of the Fourier expansions of $a, b$ and $c^{-1}$, respectively. For simplicity, we restrict ourselves to the case $a_{k}=a_{-k}, b_{k}=b_{-k}$ and $c_{k}=c_{-k}$ for all $k \in \mathbb{Z}$. Therefore, (1.4) reduces to the system

$$
a_{k}^{\prime}=f_{k}(a):=\mu b_{k}\left[a_{k}-\sum_{j+l+n=k} c_{|j|} a_{|l|} a_{|n|}\right], \quad k=0,1,2, \ldots, .
$$

The resulting finite dimensional system $f^{(m)}: \mathbb{R}^{m} \rightarrow \mathbb{R}^{m}$ upon which our numerical computations will be based is given by

$$
a_{k}^{\prime}=f_{k}^{(m)}\left(a_{0}, \ldots, a_{m-1}\right):=\mu b_{k}\left[a_{k}-\sum_{\substack{j+l+n=k \\ 0 \leq l l|,| n \mid \leq m-1}} c_{|j|} a_{|l|} a_{|n|}\right],
$$

where $k=0,1, \ldots, m-1$.

Of course, in the end we will need to be able to justify that computation with (1.6) allows us to draw conclusions about the dynamics of (1.1). With this in mind, we rewrite the form of the maps as in (1.2) and define

$$
f_{k}^{(m+)}(a):=f_{k}(a)-f_{k}^{(m)}\left(a_{0}, \ldots, a_{m-1}\right) .
$$

Then, the full system becomes

$$
a_{k}^{\prime}=f_{k}^{(m)}\left(a_{0}, \ldots, a_{m-1}\right)+f_{k}^{(m+)}(a), \quad k=0,1,2, \ldots
$$

As was indicated earlier we need to be able to bound values of the $f_{k}^{(m+)}$ term when we restrict our attention to specific compact subsets of $L^{2}$.

It is easy to check that the specific form of the nonlinear term in (1.6) comes from the fact that $\Phi$ has a quadratic nonlinearity. More generally, for a monomial of the form $c(x) a(x)^{p}$ the corresponding terms are

$$
b_{k} \sum_{n_{0}, \ldots, n_{p-1} \in \mathbb{Z}} c_{n_{0}} a_{n_{1}} \cdots a_{n_{p-1}} a_{k-\left(n_{0}+\cdots+n_{p-1}\right)} .
$$

In Section 5 we will prove the following fundamental estimates (in slightly different forms).

Proposition 1.1. Assume that there exist constants $s>1, C>0$, and $A>0$ such that

$$
\left|c_{k}\right| \leq \frac{C}{|k|^{s}} \quad \text { and } \quad\left|a_{k}\right| \leq \frac{A}{|k|^{s}}, \quad k \neq 0 .
$$

Then, for all $k \in \mathbb{Z}$,

$$
\left|\sum_{n_{0}, \ldots, n_{p-1} \in \mathbb{Z}} c_{n_{0}} a_{n_{1}} \cdots a_{n_{p-1}} a_{k-\left(n_{0}+\cdots+n_{p-1}\right)}\right| \leq\left\{\begin{array}{cc}
\frac{\alpha^{p} A^{p} C}{|k|^{s}} & k \neq 0 \\
\alpha^{p} A^{p} C & k=0
\end{array}\right.
$$


where $\alpha=\frac{2}{s-1}+2+3.5 \cdot 2^{s}$.

Proposition 1.2. Assume that there exist constants $s>1, C>0$, and $A>0$ such that

$$
\left|c_{k}\right| \leq \frac{C}{s^{|k|}} \quad \text { and } \quad\left|a_{k}\right| \leq \frac{A_{s}}{s^{|k|}}
$$

Now fix $\gamma>1$. Then, for all $k \in \mathbb{Z}$,

$$
\left|\sum_{n_{0}, \ldots, n_{p-1} \in \mathbb{Z}} c_{n_{0}} a_{n_{1}} \cdots a_{n_{p-1}} a_{k-\left(n_{0}+\cdots+n_{p-1}\right)}\right| \leq \frac{\alpha^{p} A^{p} C}{s^{|k|}} \gamma^{|k|}
$$

where $\alpha=\frac{2}{\ln s}+\frac{\gamma}{\ln (\gamma)}$.

The hypotheses of these propositions may appear artificial until one recognizes that they are regularity conditions. In particular, the assumption on $c_{k}$ is an explicit assumption on the regularity of the spatial heterogeneity of the nonlinear term in (1.1). The assumption on the $a_{k}$, however, needs greater justification. Obviously, if $a$ is a typical element of $L^{2}$, then neither (1.8) nor (1.9) will be satisfied. However, our interest lies in elements which belong to invariant sets. As was mentioned in the third observation O3, such elements often possess considerable regularity. This property will be shown explicitly in Lemma 5.1 for the Kot-Schaffer map.

To make use of Proposition 1.1 or 1.2 we need to determine the constants $A, s$, and $m$. As is explained in detail in Section 3, this is done via simulations of the finite dimensional system $f^{(L)}: \mathbb{R}^{L} \rightarrow \mathbb{R}^{L}$ where $L$ is large. In particular, we choose an initial condition $a^{0}=\left(a_{0}^{0}, \ldots, a_{L-1}^{0}\right)$ and examine the iterates $a^{1}:=f^{(L)}\left(a^{0}\right), a^{2}:=$ $f^{(L)}\left(a^{1}\right), a^{3}:=f^{(L)}\left(a^{2}\right), \ldots$. We can then divide the individual coordinates into two groups as follows. First, we look for those coordinates that fluctuate on a predetermined scale under the iterations. Typically, these involve the "lower modes," i.e. there exists an integer $m$ such that the only $a_{k}$ which change significantly under iterations of the map are those for which $k<m$. Then, one examines the remaining $a_{k}^{n}$ for $m \leq k<L$ and uses this information to choose the constants $A_{s}$ and $s$.

Observe that at this step we are not making any claims of rigor. This is an important point. Experience suggests that rigorous computations are typically expensive. Therefore, our approach is to use simulations as much as possible and at the final step to perform a rigorous verification of the results.

Using the above procedure we arrive at a finite dimensional system $f^{(m)}: \mathbb{R}^{m} \rightarrow$ $\mathbb{R}^{m}$. As was indicated earlier, the first problem we need to consider is how to find the dynamical structures of interest. From the simulation we choose a range of values of the coordinates $a_{k}, k=0, \ldots, m-1$, i.e. we choose constants $a_{k}^{-}$and $a_{k}^{+}$such that we are only interested in $a_{k} \in \widetilde{a}_{k}:=\left[a_{k}^{-}, a_{k}^{+}\right]$. Let

$$
W:=\prod_{k=0}^{m-1}\left[a_{k}^{-}, a_{k}^{+}\right]
$$

and consider $f^{(m)}: W \rightarrow \mathbb{R}^{m}$.

Having fixed $W$, we can now determine the constant $A$ in Proposition 1.1 or 1.2. Observe that by this procedure we have effectively restricted our attention to the dynamics of $\Phi$ on the set

$$
Z:=W \times \prod_{k=m}^{\infty} \widetilde{a}_{k}
$$


where $\widetilde{a}_{k}:=\left[-\frac{A_{s}}{|k|^{s}}, \frac{A_{s}}{|k|^{s}}\right]$ or $\widetilde{a}_{k}:=\left[-\frac{A_{s}}{s^{|k|}}, \frac{A_{s}}{s^{|k|}}\right]$ depending on whether one assumes polynomial or exponential decay rates. Clearly, for $s$ sufficiently large, $Z$ is a compact set. The obvious question at this point is whether this assumption restricts the types of invariant sets that one can capture. Here again the third observation $\mathbf{O 3}$ comes into play. For a large variety of systems elements of invariant sets possess significant regularity properties, and therefore, will lie in a set of the form of $Z$.

We can then use Proposition 1.1 or 1.2 to determine bounds $\varepsilon_{k}^{(m+)}>0$ for $f_{k}^{(m+)}$, $k=0,1,2, \ldots$, i.e. for all $a \in Z,\left|f_{k}^{(m+)}(a)\right|<\varepsilon_{k}^{(m+)}$.

1.2. Analysis of Finite Dimensional Systems. Up to this point in the analysis, most of what has been described is easily subsumed within the standard Galerkin approximation techniques. We now change the perspective in our analysis of the finite dimensional system. While we have an explicit representation of $f_{k}^{(m)}$, we are really interested in the dynamics of the system generated by the $f_{k}$. Unfortunately, the best we have been able to do is to determine a bound $\varepsilon_{k}^{(m+)}$ on $f_{k}^{(m+)}$. To incorporate the errors into our analysis we adopt the philosophy that our finite dimensional system should be viewed as a set valued map satisfying (1.3).

Observe that even if the multivalued map satisfies (1.3) it is probably not directly amenable to analysis by the computer, since it does not have a combinatorial structure. To resolve this problem we partition $W$ into a cubical grid

$$
\mathcal{G}^{(\sigma)}:=\left\{G=\prod_{k=0}^{m-1} a_{k}^{-}+\left[\frac{i_{k}}{2^{\sigma}}, \frac{i_{k}+1}{2^{\sigma}}\right] w_{k} \mid i_{k} \in\left\{0, \ldots, 2^{\sigma}-1\right\}\right\}
$$

where $w_{k}=a_{k}^{+}-a_{k}^{-}$and $\sigma \in \mathbb{N}$. Given $\mathcal{B} \subset \mathcal{G}^{(\sigma)}$, let $|\mathcal{B}|$ denote the union of all the elements in $\mathcal{B}$ viewed as subsets of $\mathbb{R}^{m}$. We will use a multivalued map $\mathcal{F}^{(\sigma)}: \mathcal{G}^{(\sigma)} \rightrightarrows \mathcal{G}^{(\sigma)}$ that satisfies the following property. For every $G \in \mathcal{G}^{(\sigma)}$

$$
\left\{\left(f_{0}, \ldots, f_{m-1}\right)(a) \mid a \in G \times \prod_{k=m}^{\infty} \widetilde{a}_{k}\right\} \subset \operatorname{int}\left(\left|\mathcal{F}^{(\sigma)}(G)\right|\right) .
$$

Observe that this is just a restatement of (1.3).

For the moment we will treat $\sigma$ as fixed and so simplify the notation by setting $\mathcal{F}=\mathcal{F}^{(\sigma)}$ and $\mathcal{G}=\mathcal{G}^{(\sigma)}$. Since $\mathcal{F}$ is defined on the set of cubes in $\mathcal{G}$ and has images that consist of sets of these cubes, it is a combinatorial object. Hence it can be analyzed directly by the computer. A precise description of the construction of $\mathcal{F}$ is given in Section 3. For now it is sufficient to observe that it is determined by the bounds $\varepsilon_{k}^{(m+)}$ on $f_{k}^{(m+)}$ and estimates on $f_{k}^{(m)}$ for $k=0, \ldots, m-1$. Furthermore, the estimates on $f_{k}^{(m)}$ depend on the size of the elements of $\mathcal{G}$. In particular, larger $\sigma$ leads to better estimates.

$\mathcal{F}$ is the finite dimensional dynamical system whose structure we will study. Since the reader may not have encountered multivalued dynamical systems before, we include a few essential definitions at this point. A full combinatorial trajectory of $\mathcal{F}$ through $G \in \mathcal{G}$ is a bi-infinite sequence $\gamma_{G}: \mathbb{Z} \rightarrow \mathcal{G}$ satisfying: $\gamma_{G}(0)=G$ and $\gamma_{G}(n+1) \in \mathcal{F}\left(\gamma_{G}(n)\right)$ for all $n \in \mathbb{Z}$. $\mathcal{S} \subset \mathcal{G}$ is a combinatorial invariant set if for every $G \in \mathcal{S}$ there exists a full solution $\gamma_{G}: \mathbb{Z} \rightarrow \mathcal{S}$.

Even though $\mathcal{F}$ is a finite combinatorial object, and therefore, capable of being examined directly by the computer, the associated complexity is impractical. Observe that the number of elements of $\mathcal{G}$ is $\left(2^{\sigma}\right)^{\operatorname{dim} W}$. To avoid this problem we make use of a 
subdivision algorithm as described in [4] to find the maximal invariant set $\mathcal{A}$ of $\mathcal{F}$ in $\mathcal{G}$. The computational effort to find $\mathcal{A}$ is of the same order as the number of elements in $\mathcal{A}$ which is approximately $\left(2^{\sigma}\right)^{\operatorname{dim} \mathcal{A}}$. This is the point at which the second observation O2 comes into play. If we are looking for low dimensional dynamical structures, then we can hope to do so in a computationally efficient manner even if $m$, the dimension of the approximation, is large.

The condition (1.11) guarantees that $\mathcal{F}$ acts as an outer approximation of the map $f^{(m)}$. However, knowledge of trajectories of $\mathcal{F}$ does not directly lead to information about the existence of trajectories of $f^{(m)}$. For example, the existence of a fixed point for $\mathcal{F}$, i.e. a full solution $\gamma_{G}: \mathbb{Z} \rightarrow \mathcal{G}$ that has the form $\gamma_{G}(n)=G$ for all $n \in \mathbb{Z}$, does not imply that $f^{(m)}$ possesses a fixed point. To obtain this information requires the use of algebraic topology and, in particular, the Conley index theory.

The starting point for the computational version of this theory is the notion of a combinatorial isolating neighborhood, a finite set whose maximal invariant set lies strictly in its interior. To make this precise in the setting of a cubical grid, given $\mathcal{B} \subset \mathcal{G}$, let

$$
o(\mathcal{B}):=\{G \in \mathcal{G}|| G|\cap| \mathcal{B} \mid \neq \emptyset\} .
$$

Observe that $o(\mathcal{B})$ is the smallest neighborhood of $\mathcal{B}$ that can be represented using elements of the cubical grid. Given $\mathcal{I} \subset \mathcal{G}$, the maximal combinatorial invariant set in $\mathcal{I}$ is

$$
\operatorname{Inv}(\mathcal{I}, \mathcal{F}):=\left\{G \in \mathcal{I} \mid \text { there exists a full trajectory } \gamma_{G}: \mathbb{Z} \rightarrow \mathcal{I}\right\}
$$

$\mathcal{I}$ is a combinatorial isolating neighborhood if

$$
o(\operatorname{Inv}(\mathcal{I})) \subset \mathcal{I} .
$$

An important result following from (1.11) is that if $\mathcal{I}$ is an isolating neighborhood for $\mathcal{F}$, then $|\mathcal{I}|$ is an isolating neighborhood for $f^{(m)}$.

To each isolating neighborhood $I$ of $f^{(m)}$ one can assign a Conley index which is denoted by $\chi_{*}\left(I, f^{(m)}\right)$. To compute this index one needs to first construct an index pair. As was indicated earlier these computations are done using the combinatorial multivalued map for which we make use of the following definition.

Definition 1.3. Let $\mathcal{I}$ be an isolating neighborhood for $\mathcal{F}$. A pair $\mathcal{N}=\left(\mathcal{N}_{1}, \mathcal{N}_{0}\right)$ of subsets $\mathcal{N}_{0} \subset \mathcal{N}_{1} \subset \mathcal{I}$ is a combinatorial index pair if the following conditions are satisfied:

1. $\mathcal{F}\left(\mathcal{N}_{i}\right) \cap \mathcal{I} \subset \mathcal{N}_{i}$

2. $\mathcal{F}\left(\mathcal{N}_{1} \backslash \mathcal{N}_{0}\right) \subset \mathcal{I}$

3. $o(\operatorname{Inv}(\mathcal{I}, \mathcal{F})) \subset \mathcal{N}_{1} \backslash \mathcal{N}_{0}$.

Of course our immediate interest does not lie with the multivalued map $\mathcal{F}$, but rather with the single valued map $f^{(m)}$. We will make use of the fact that an index pair for $\mathcal{F}$ is also an index pair for $f^{(m)}$. In fact, Szycmzak [22, 23] has shown that we can use a slightly weaker construction based on $\mathcal{F}$. Let $\mathcal{S}$ be an isolated invariant set for $\mathcal{F}$. Define,

$$
\mathcal{N}_{1}:=\mathcal{S} \cup \mathcal{F}(\mathcal{S}) \quad \text { and } \quad \mathcal{N}_{0}:=\mathcal{N}_{1} \backslash \mathcal{S} .
$$

Then, $|\mathcal{N}|=\left(\left|\mathcal{N}_{1}\right|,\left|\mathcal{N}_{0}\right|\right)$ is an index pair for $f^{(m)}$.

Further details concerning this index are provided in Section 2. For the moment two remarks suffice. The first is that $\chi_{*}\left(\left|\mathcal{N}_{1}\right| \backslash\left|\mathcal{N}_{0}\right|, f^{(m)}\right)$, the Conley index under 
$f^{(m)}$, can be computed using the combinatorial information of $\mathcal{F}$. The second is that the Conley index provides information about the structure of the associated maximal invariant set.

These remarks should make it clear that in order to use the Conley index to understand the dynamics of $f^{(m)}$ it is essential that we are able to efficiently find isolating neighborhoods $\mathcal{I}$ of $\mathcal{F}$. Furthermore, we need to be able to find isolating neighborhoods that isolate specific dynamical objects of interest such as fixed points, periodic orbits, heteroclinic orbits, etc. Up to this point we have treated $\mathcal{F}$ as a dynamical system. However, to efficiently find specified orbits, it is useful to view $\mathcal{F}$ as a directed graph; the vertices correspond to the elements of $\mathcal{G}$, and if $G_{1} \in \mathcal{F}\left(G_{0}\right)$ then there is a directed edge from $G_{0}$ to $G_{1}$. Full trajectories of $\mathcal{F}$ now correspond to infinite paths in the directed graph. These paths or sets of paths, representing either recurrent sets or connecting orbits between recurrent sets, can be found using standard graph theoretic algorithms (see [7] and references therein). In this paper we make use of the implementation in the software package GAIO as detailed in [5].

Observe that the above mentioned paths correspond to a set of elements $\mathcal{I} \subset \mathcal{G}$. $\mathcal{I}$ is clearly an invariant set for $\mathcal{F}$, and hence, cannot be an isolating neighborhood. However, $o(\mathcal{I})$ is a candidate to be an isolating neighborhood. In particular, we can compute $\operatorname{Inv}(o(\mathcal{I}), \mathcal{F})$. If $o(\operatorname{Inv}(o(\mathcal{I}), \mathcal{F})) \subset \mathcal{I}$, then $\mathcal{I}$ is an isolating neighborhood. If not, then we can repeat the procedure starting with the larger invariant set $\operatorname{Inv}(o(\mathcal{I}), \mathcal{F})$. The specific algorithm is given in Section 4 .

1.3. Lifting to the Full System. At this point we have described the finite dimensional reduction and the computer assisted analysis of the resulting finite dimensional system. In particular, assume that we have found a pair of subsets $\mathcal{N}=\left(\mathcal{N}_{1}, \mathcal{N}_{0}\right)$ of $\mathcal{G}$ satisfying (1.13). As was mentioned above, this allows us to determine the Conley index $\chi_{*}\left(\left|\mathcal{N}_{1}\right| \backslash\left|\mathcal{N}_{0}\right|, f^{(m)}\right)$. This is the information that we wish to lift to the full infinite dimensional system.

Recall that we have restricted our attention to the dynamics in

$$
Z=W \times \prod_{k=m}^{\infty} \tilde{a}_{k}
$$

Let us further assume that for high modes, i.e. $k \geq m$, the system is contracting. More precisely, assume that for all $a \in Z$

$$
f_{k}(a) \subset \operatorname{int}\left(\widetilde{a}_{k}\right)
$$

In practice this is verified using Proposition 1.1 or 1.2 .

In this case it is easy to show (see Theorem 2.3) that

$$
\widetilde{N}=\left(\widetilde{N}_{1}, \widetilde{N}_{0}\right):=\left(\left|\mathcal{N}_{1}\right| \times \prod_{k=m}^{\infty} \widetilde{a}_{k},\left|\mathcal{N}_{0}\right| \times \prod_{k=m}^{\infty} \widetilde{a}_{k}\right)
$$

is an index pair for $\Phi$ and that

$$
\chi_{*}\left(\widetilde{N}_{1} \backslash \widetilde{N}_{0}, \Phi\right) \cong \chi_{*}\left(\left|\mathcal{N}_{1}\right| \backslash\left|\mathcal{N}_{0}\right|, f^{(m)}\right)
$$

Observe that both $\widetilde{N}_{1}$ and $\widetilde{N}_{0}$ are compact sets. Therefore, we can apply the classical results from the Conley index theory to draw conclusions about the dynamics of $\Phi$. 
1.4. Refinement of the combinatorial invariant set. We mentioned above that given an isolating neighborhood, we have algorithms that compute the associated Conley index. As will become clear when we present the results from specific computations in Section 6, this is by far the most computationally intensive step. Therefore, our strategy is to compute the Conley index using a minimal number of modes, i.e. as small an $m$ as possible, and the fewest boxes, i.e. choosing $\sigma$ as small as possible.

As was described earlier, we use the Conley index of an isolating neighborhood $I$ to obtain information about $\operatorname{Inv}\left(I, f^{(m)}\right)$. Since $\operatorname{Inv}\left(I, f^{(m)}\right) \subset I$, the diameters of the components of $I$ provide us with bounds on the individual trajectories in $\operatorname{Inv}\left(I, f^{(m)}\right)$. One of the other important properties of the Conley index is that it is an index of invariant sets, i.e. if $I$ and $I^{\prime}$ are different isolating neighborhoods such that

$$
\operatorname{Inv}\left(I, f^{(m)}\right)=\operatorname{Inv}\left(I^{\prime}, f^{(m)}\right)
$$

then $\chi_{*}\left(I, f^{(m)}\right)=\chi_{*}\left(I^{\prime}, f^{(m)}\right)$. This allows us to efficiently improve the bounds on the invariant sets as follows. Let $\mathcal{I} \subset \mathcal{G}^{(\sigma)}$ be an isolating neighborhood for the map $\mathcal{F}^{(\sigma)}$. Now choose $\sigma^{\prime}>\sigma$. Then, there exists a set $\mathcal{B} \subset \mathcal{G}^{\left(\sigma^{\prime}\right)}$ such that $|\mathcal{B}|=|\mathcal{I}|$. Let $\mathcal{I}^{\prime}:=\operatorname{Inv}\left(\mathcal{B}, \mathcal{F}^{\left(\sigma^{\prime}\right)}\right)$. Then, in general $\left|\mathcal{I}^{\prime}\right| \subset|\mathcal{I}|$. However, $\chi_{*}\left(\left|\mathcal{I}^{\prime}\right|\right)=\chi_{*}(|\mathcal{I}|)$. Thus, we retain the information about the invariant set, but with better bounds.

Still there is a natural limit to how big we can choose $\sigma^{\prime}$ for a given $m$. This limit is essentially determined by the bounds $\varepsilon_{k}^{(m+)}$ on the terms $f_{k}^{(m+)}$ in $(1.7)$ - note that one would not expect $\left|\mathcal{I}^{\prime}\right|$ to be any smaller than $|\mathcal{I}|$ if the bounds $\varepsilon_{k}^{(m+)}$ are of the same order of magnitude as the size of the grid elements in $G^{\left(\sigma^{\prime}\right)}$. There are two ways to escape from this dilemma:

1. Improve the values $a_{k}^{-}, a_{k}^{+}, k \geq m$, i.e. make the intervals $\left[a_{k}^{-}, a_{k}^{+}\right]$as small as possible - since this will decrease the bounds $\varepsilon_{k}^{(m)}$ via Propositions 1.1 and 1.2. Once a tighter neighborhood $\mathcal{I}^{\prime}$ has been constructed, the intervals can be updated such that (1.14) is still satisfied.

2. Increase the number of modes $m$ used in the computations - this will also decrease the bounds $\varepsilon_{k}^{(m+)}$. Again we make use of the fact that by suitably lifting the isolating neighborhood $\mathcal{I}$ from $\mathbb{R}^{m}$ to $\mathbb{R}^{m+1}$, we retain the index information and thus the information about the invariant set. One may think that the computational effort increases dramatically when going to higher dimensional phase spaces. This is not the case as will be detailed in later sections and demonstrated in the examples section. In fact, due to the hierachical design of the refinement process and the way the combinatorial map $\mathcal{F}$ is computed, the computational effort grows only linearly in $m$.

1.5. Results. In Section 6 we are going to prove a couple of prototype results about the dynamics of the map $\Phi$ for different parameter values. These results are meant to serve as examples of what kind of statements may be obtained by our method and are, of course, by no means a complete description of the dynamics of $\Phi$. We are going to sketch these results in the following, for the exact technical statements we refer to Section 6.

RESULT 1. For certain parameter values the map $\Phi$ possesses a fixed point $a_{1}$ and a period two point $a_{2}$, as well as an orbit limiting in backward time to a neighborhood of $a_{1}$ and in forward time to a neighborhood of $a_{2}$. We localize these objects up to an error of $10^{-12}$.

RESULT 2. For the same parameter values the map $\Phi$ possesses an invariant set on which it exhibits complicated dynamics. The topological entropy of $\Phi$ is at least 


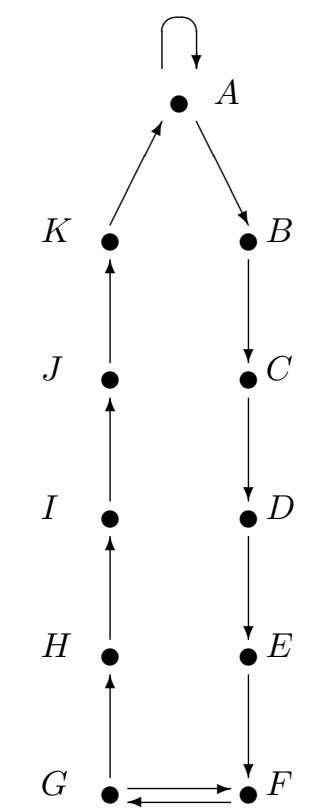

FIG. 1.1. The graph $T$.

1.2 .

RESULT 3. For certain parameter values the map $\Phi$ possesses an invariant set on which $\Phi$ is semi-conjugate to the subshift dynamics given by the transition graph T shown in Figure 1.1.

2. The Conley Index. The Conley index theory is a key component in our approach to the rigorous analysis of high dimensional dynamical systems. It provides the tool by which we can pass from combinatorial data to dynamical structures. We present it here (for the most part) as a black box; that is notation, definitions and key theorems are stated, but no attempt is made to motivate the underlying justification. In part this is done for reasons of space, but also to convey the fact that the computational methods being developed are sufficiently self contained that they can be performed without knowledge of the index theory and similarly the index theory can be applied without reference to the details of the computations. As such, depending on the reader's preferences, this section can be skipped and only referred to for notation or critical results.

There are two issues that in the context of our approach are of particular importance. The first is robustness of the index with respect to perturbation. There are essentially two perturbations that need to be controlled: the numerical approximation used to study the finite dimensional map and the finite dimensional approximation of the true infinite dimensional dynamics. The second issue is that of using the index to recover information about the dynamics.

2.1. Robustness. As was indicated in the Introduction, depending on our immediate needs we use the original system to construct three types of dynamical systems in this paper: continuous maps, $f: Y \rightarrow Y^{\prime}$; multivalued maps, $F: Y \rightrightarrows Y^{\prime}$; and combinatorial maps, $\mathcal{F}: \mathcal{G} \rightrightarrows \mathcal{G}$. It is assumed that $Y$ is a compact subset of $Y^{\prime}$ and that $\mathcal{G}$ is a cubical complex such that $|\mathcal{G}|=Y$.

Of course, the relationship between these maps is crucial. Throughout our discussion we require that $(1.11)$ be satisfied. Observe that the process of passing from a combinatorial map to a multivalued map is quite simple. Given $\mathcal{F}$, define $F:|\mathcal{G}| \rightrightarrows|\mathcal{G}|$ by

$$
F(y):=\bigcup_{y \in G}|\mathcal{F}(G)|
$$


Now, observe that a single valued map $f: Y \rightarrow Y$ is a special case of a multivalued map $F: Y \rightrightarrows Y$. Therefore, any definition given in terms of a multivalued map is immediately applicable to continuous maps. In the Introduction we gave some of the fundamental definitions in the setting of combinatorial dynamics. We repeat them now in the context of multivalued maps.

A full trajectory of $F$ is a bi-infinite sequence $\sigma_{y}: \mathbb{Z} \rightarrow Y$ satisfying: $\sigma_{y}(0)=y$ and $\sigma_{y}(n+1) \in F\left(\sigma_{y}(n)\right)$ for all $n \in \mathbb{Z}$. $S \subset Y$ is an invariant set if for every $y \in S$ there exists a full solution $\sigma_{y}: \mathbb{Z} \rightarrow S$. A compact set $I \subset Y$ is an isolating neighborhood if its maximal invariant set is contained in its interior, i.e.

$$
\operatorname{Inv}(I, F):=\left\{y \in I \mid \exists \sigma_{y}: \mathbb{Z} \rightarrow I\right\} \subset \operatorname{int}(I) .
$$

An isolated invariant set is the maximal invariant set of an isolating neighborhood.

The following definition is due to Szymczak in [23].

Definition 2.1. A pair $N=\left(N_{1}, N_{0}\right)$ of compact sets is an index pair for $F$ if the following conditions are satisfied:

1. $F\left(N_{0} \cap \operatorname{cl}\left(N_{1} \backslash N_{0}\right)\right) \cap N_{1} \subset N_{0}$;

2. $F\left(N_{1} \backslash N_{0}\right) \subset N_{1}$;

3. $I:=\operatorname{cl}\left(N_{1} \backslash N_{0}\right)$ is an isolating neighborhood under $F$

Notice that if one applies the same idea used to transform the combinatorial map $\mathcal{F}$ to the multivalued map $F$ as in (2.1), then the definitions for the combinatorial objects given in the introduction are consistent with these new definitions. In this way we can use graph theoretic techniques to compute topological objects.

Let $F, F^{\prime}: Y \rightrightarrows Y^{\prime} . F$ encloses $F^{\prime}$ if

$$
F^{\prime}(y) \subset F(y)
$$

for every $y \in Y$. In the special case that $F^{\prime}$ is a continuous single valued map, $F^{\prime}$ is called a continuous selector for $F$.

The following result is trivial, but is of special interest in the case that $F^{\prime}$ is a continuous selector, because it allows us to transfer the topological constructions for multivalued maps (which encode the errors) to continuous maps which are not explicitly known.

Proposition 2.2. Assume $F$ encloses $F^{\prime}$.

1. If $\sigma_{y}$ is a trajectory for $F^{\prime}$, then $\sigma_{y}$ is a trajectory for $F$.

2. If $I$ is an isolating neighborhood for $F$, then $I$ is an isolating neighborhood for $F^{\prime}$.

3. If $N=\left(N_{1}, N_{0}\right)$ is an index pair for $F$, then $N$ is an index pair for $F^{\prime}$.

Now consider the case of a continuous map $f: Y \rightarrow Y^{\prime}$. Let $N=\left(N_{1}, N_{0}\right)$ be an index pair under $f$ with isolating neighborhood $I:=\operatorname{cl}\left(N_{1} \backslash N_{0}\right)$. Let $N_{1} / N_{0}$ denote the quotient space obtained by identifying the elements of $N_{0}$ to a single point. Then, by [23, Theorem 1.4] $f$ induces a continuous map

$$
f_{N}:\left(N_{1} / N_{0},\left[N_{0}\right]\right) \rightarrow\left(N_{1} / N_{0},\left[N_{0}\right]\right) .
$$

On the level of homology, $f_{N}$ induces a map

$$
\chi_{*}(I, f): H_{*}\left(N_{1}, N_{0}\right) \rightarrow H_{*}\left(N_{1}, N_{0}\right)
$$

which, up to an equivalence class, is the Conley index of $I$. For details the reader is referred to $[23,8,16]$. 
A fundamental property of the Conley index which we will exploit heavily is the following. Let $I$ be an isolating neighborhood for a multivalued map $F$ taking contractible values. If $g$ and $f$ are continuous selectors for $F$, then their Conley indices are the same, i.e.

$$
\chi_{*}(I, f) \cong \chi_{*}(I, g) .
$$

Since, $F$ encodes the numerical errors, this is a statement about the robustness of the index with respect to numerical error.

We now have the following sequence of implications. Let $\mathcal{F}$ be a combinatorial map. As will be indicated in Section 3 there are efficient algorithms to produce an isolating neighborhood $\mathcal{I}$ and index pair $\mathcal{N}=\left(\mathcal{N}_{1}, \mathcal{N}_{0}\right)$ for $\mathcal{F}$. Using (2.1) we obtain a multivalued map $F$ for which $I=|\mathcal{I}|$ is an isolating neighborhood and $N=\left(N_{1}, N_{0}\right)=\left(\left|\mathcal{N}_{1}\right|,\left|\mathcal{N}_{0}\right|\right)$ is an index pair. Let $f$ be a continuous selector for $F$. Then, $I$ is an isolating neighborhood for $f$ with an index pair $N=\left(N_{1}, N_{0}\right)$. An important remark is that one can use the combinatorial information of $\mathcal{F}$ and $\mathcal{N}$ to compute $H_{*}\left(N_{1}, N_{0}\right)$ and $\chi_{*}(I, f)$, see $[17,11]$.

We now focus our attention to a specific setting of relevance to this paper. Let $X$ be a Hilbert space with an orthogonal basis $\left(\varphi_{k}\right)$. Let $P_{m}: X \rightarrow X_{m}:=$ $\operatorname{span}\left\{\varphi_{k} \mid k=0, \ldots, m\right\}$ be a projection and let $Q_{m}=I-P_{m}$ be the projection onto the complementary subspace $X_{m}^{\prime}$.

Let $W \subset X_{m}$ and $V \subset X_{m}^{\prime}$ be compact subsets. In addition, assume that $V$ is contractible. Let $\partial V$ denote the boundary of $V \subset X_{m}^{\prime}$ and let $Z=W \times V$.

THEOREM 2.3. Let $F^{\prime}: Z \rightrightarrows X_{m} \times V$ and $F: W \rightrightarrows X_{m}$ be a multivalued maps such that

$$
P_{m} F^{\prime}(z) \subset F\left(P_{m} z\right) \quad \forall z \in Z
$$

Assume that

$$
Q_{m} F^{\prime}(Z) \subset \operatorname{int}(V) .
$$

Then the following results hold:

(a) If $I$ is an isolating neighborhood for $F$, then $\hat{I}:=I \times V$ is an isolating neighborhood for $F^{\prime}$.

(b) If $N=\left(N_{1}, N_{0}\right)$ is an index pair for $F$, then $\hat{N}:=\left(N_{1} \times V, N_{0} \times V\right)$ is an index pair for $F^{\prime}$.

(c) Let $g$ and $g^{\prime}$ be continuous selectors for $F$ and $F^{\prime}$, respectively, then

$$
\chi_{*}\left(\hat{I}, g^{\prime}\right) \cong \chi_{*}(I, g) .
$$

Proof. (a) To show that $\hat{I}$ is an isolating neighborhood it is sufficient to show that $\partial(\hat{I}) \cap \operatorname{Inv}\left(\hat{I}, F^{\prime}\right)=\emptyset$. Consider $z \in \partial(\hat{I})$. Suppose first that $P_{m} z \in \partial I$ and let $\sigma_{z}: \mathbb{Z} \rightarrow X$ be a full trajectory through $z$ under $F^{\prime}$, i.e. $\sigma_{z}(n+1) \in F^{\prime}\left(\sigma_{z}(n)\right)$ and $\sigma_{z}(0)=z$. Observe that by $(2.2), P_{m} \sigma_{z}$ defines a full trajectory for $F$. However, $P_{m} z \in \partial I$. Since $I$ is an isolating neighborhood for $F$, there exists $n \in \mathbb{Z}$, such that $P_{m} \sigma_{z}(n) \notin I$ and hence $\sigma_{z}(n) \notin \hat{I}$. If, however, $P_{m} z \notin \partial I$ then $Q_{m} z \in \partial V$. In this second case, (2.3) implies that $z \notin \operatorname{Inv}\left(\hat{I}, F^{\prime}\right)$.

(b) Suppose $N=\left(N_{1}, N_{0}\right)$ is an index pair for $F$. We now prove that $\hat{N}:=$ $\left(\hat{N}_{1}, \hat{N}_{0}\right)$, where $\hat{N}_{i}:=N_{i} \times V$, is an index pair for $F^{\prime}$. By Definition 2.1(3), I := $\operatorname{cl}\left(N_{1} \backslash N_{0}\right)$ is an isolating neighborhood under $F$. By (a), $\hat{I}:=\operatorname{cl}\left(\hat{N}_{1} \backslash \hat{N}_{0}\right)$ is an 
isolating neighborhood for $F^{\prime}$ and condition (3) is satisfied for the pair $\hat{N}$. We now need to check that the remaining two conditions of Definition 2.1 are satisfied by $\hat{N}=\left(\hat{N}_{1}, \hat{N}_{0}\right)$.

(1) Let $z \in \hat{N}_{0} \cap \operatorname{cl}\left(\hat{N}_{1} \backslash \hat{N}_{0}\right)$. Then $P_{m} z \in N_{0} \cap \operatorname{cl}\left(N_{1} \backslash N_{0}\right)$ and using (2.2),

$$
P_{m}\left(F^{\prime}(z) \cap \hat{N}_{1}\right) \subset F\left(P_{m} z\right) \cap N_{1} \subset N_{0}=P_{m}\left(\hat{N}_{0}\right)
$$

By (2.3),

$$
Q_{m}\left(F^{\prime}(z) \cap \hat{N}_{1}\right)=Q_{m}\left(F^{\prime}(z)\right) \cap V \subset Q_{m}\left(\hat{N}_{0}\right)
$$

Therefore, $F^{\prime}\left(\hat{N}_{0} \cap \operatorname{cl}\left(\hat{N}_{1} \backslash \hat{N}_{0}\right)\right) \cap \hat{N}_{1} \subset \hat{N}_{0}$.

(2) Let $z \in \hat{N}_{1} \backslash \hat{N}_{0}$. By $(2.2), P_{m} F^{\prime}(z) \subset F\left(P_{m} z\right)$. Since $P_{m} z \in N_{1} \backslash N_{0}$, $F\left(P_{m} z\right) \subset N_{1}=P_{m} \hat{N}_{1}$. Hence, $P_{m} F^{\prime}(z) \subset P_{m} \hat{N}_{1}$. In addition, by (2.3) $Q_{m} F^{\prime} z \subset$ int $V \subset Q_{m} \hat{N}_{1}$. Therefore, $F^{\prime}\left(\hat{N}_{1} \backslash \hat{N}_{0}\right) \subset \hat{N}_{1}$.

(c) By assumption $V$ is contractible, i.e. there exists a continuous map $h: V \times$ $[0,1] \rightarrow V$, such that $h(\cdot, 0)=I_{V}$, the identity on $V$, and $h(\cdot, 1)=v_{0} \in V$. Define $H: Z \times[0,1] \rightarrow Z$ by $H(z, s)=P_{m} g^{\prime}(z)+h\left(Q_{m} g^{\prime}(z), s\right)$. Observe that $H(z, 0)=g^{\prime}(z)$ and $H(\cdot, 1): Z \rightarrow W \times\left\{v_{0}\right\}$. Furthermore,

$$
P_{m} H(z, s) \in F\left(P_{m} z\right) \quad \forall z \in Z .
$$

Therefore, if $\hat{I}$ is an isolating neighborhood for $F^{\prime}$ then it is also an isolating neighborhood for $H(\cdot, s)$ for all $s \in[0,1]$. By the continuation property of the Conley index, $\chi_{*}(\hat{I}, H(\cdot, 1)) \cong \chi_{*}\left(\hat{I}, g^{\prime}\right)$.

Define $q: W \rightarrow X_{m}$ by $q(w)=P_{m} H\left(w+v_{0}, 1\right)$. By [19, Theorem 1.12], $\chi_{*}(I, q) \cong$ $\chi_{*}(\hat{I}, H(\cdot, 1))$. Finally, the result follows by [12, Theorem 5.4].

The following two corollaries which are just special cases of the previous theorem are used in this paper. The first allows us to lift the information from $\mathbb{R}^{m}$ to $\mathbb{R}^{m+1}$. As was indicated in the Introduction computing the homology index is by far the most expensive calculation and the expense grows rapidly with dimension. Therefore, we adopt the strategy of computing the index with a very coarse low dimensional approximation, i.e. $\chi_{*}(I, g)$ in the following corollary. To improve the accuracy we need to increase the dimension of the approximation. This corollary guarantees that the index does not change.

Corollary 2.4. Let $F^{\prime}: W \times\left[a^{-}, a^{+}\right] \rightrightarrows X_{m} \times\left[a^{-}, a^{+}\right]$and $F: W \rightrightarrows X_{m}$ be multivalued maps satisfying (2.2). Let $F_{m}^{\prime}(z):=Q_{m} F^{\prime}(z)$ and assume that $F_{m}^{\prime}(Z) \subset$ $\left(a^{-}, a^{+}\right)$.

(a) If $I$ is an isolating neighborhood for $F$, then $\hat{I}:=I \times\left[a^{-}, a^{+}\right]$is an isolating neighborhood for $F^{\prime}$.

(b) If $N=\left(N_{1}, N_{0}\right)$ is an index pair for I under $F$, then $\hat{N}:=\left(N_{1} \times\left[a^{-}, a^{+}\right], N_{0} \times\right.$ $\left.\left[a^{-}, a^{+}\right]\right)$is an index pair for $\hat{I}$ under $F^{\prime}$.

(c) Let $g$ and $g^{\prime}$ be continuous selectors for $F$ and $F^{\prime}$, respectively, then

$$
\chi_{*}\left(\hat{I}, g^{\prime}\right) \cong \chi_{*}(I, g) .
$$

Once the dimension of the approximation has been increased sufficiently to obtain the desired accuracy, the following result implies that the index computed with the finite dimensional approximation is actually valid for the full infinite dimensional system. We now return to the notation of the Introduction. In particular, as in (1.10) 
$Z=W \times \prod_{k=m}^{\infty} \widetilde{a}_{k}$ where $\widetilde{a}_{k}:=\left[-\frac{A_{s}}{|k|^{s}}, \frac{A_{s}}{|k|^{s}}\right]$ or $\widetilde{a}_{k}:=\left[-\frac{A_{s}}{s^{|k|}}, \frac{A_{s}}{\left.s^{|k|}\right]}\right.$. Furthermore, $s$ is choosen large enough that $Z$ is compact. Also, as in (1.5), $a_{k}^{\prime}=f_{k}(a)$ for all $a \in Z$.

Corollary 2.5. Let $f: Z \rightarrow X_{m} \times V$ be a continuous map. Let $F: W \rightrightarrows \mathbb{R}^{m}$ be a multivalued map satisfying

$$
P_{m} f(a) \subset F\left(P_{m} a\right) \quad \forall a \in Z .
$$

Finally, assume that for all $k \geq m$ and all $a \in Z$

$$
f_{k}(a) \in\left(a_{k}^{-}, a_{k}^{+}\right) .
$$

(a) If $I$ is an isolating neighborhood for $F$, then $\hat{I}:=I \times V$ is an isolating neighborhood for $f$.

(b) If $N=\left(N_{1}, N_{0}\right)$ is an index pair for I under $F$, then $\hat{N}:=\left(N_{1} \times V, N_{0} \times V\right)$ is an index pair for $\hat{I}$ under $f$.

(c) If $g$ is continuous selector for $F$, then

$$
\chi_{*}(\hat{I}, f) \cong \chi_{*}(I, g) .
$$

2.2. Dynamics via the Conley Index. We now turn to the question of how the Conley index can be used to draw conclusions about the dynamics of a continuous map $f$. For a particularly clear and complete explanation the reader is referred to [23] which is the basis for the following discussion. Let $N=\left(N_{1}, N_{0}\right)$ be an index pair for an isolating neighborhood $I$ of $f$. Let $\left(N_{1} / N_{0},\left[N_{0}\right]\right)$ be the pointed topological space obtained by collapsing $N_{0}$ to a single point. Since $N=\left(N_{1}, N_{0}\right)$ is an index pair, the induced quotient map

$$
f_{N}:\left(N_{1} / N_{0},\left[N_{0}\right]\right) \rightarrow\left(N_{1} / N_{0},\left[N_{0}\right]\right)
$$

is continuous, and hence defines a dynamical system on a compact set. In particular, we have the induced map on homology

$$
f_{N *}: H_{*}\left(N_{1} / N_{0},\left[N_{0}\right]\right) \rightarrow H_{*}\left(N_{1} / N_{0},\left[N_{0}\right]\right) .
$$

We will write

$$
f_{N, n}: H_{n}\left(N_{1} / N_{0},\left[N_{0}\right]\right) \rightarrow H_{n}\left(N_{1} / N_{0},\left[N_{0}\right]\right)
$$

when we need to indicate the homology map on the $n$-th level.

Since in our applications the $N_{i}$ are polygons, $f_{N *}$ is equivalent to the Conley index map $\chi_{*}(I, f)$. For the same reason, throughout this section we will assume that $H_{*}\left(N_{1}, N_{0}\right)$ are free abelian groups, in other words the relative homology groups of the index pairs do not have a torsion subgroup.

The simplest dynamical result is the Ważewski principle: if $f_{N *}$ is not nilpotent, then $\operatorname{Inv}(I, f) \neq \emptyset$. Under appropriate conditions it is also fairly simple to check for the existence of a fixed point. Let

$$
\Lambda(I, f):=\sum_{n=0}^{\infty}(-1)^{n} \operatorname{tr} f_{N, n}
$$

and if $\Lambda(I, f) \neq 0$, then $f$ has a fixed point in $I$ [23, Corollary 1.2]. Similarly, if $\Lambda\left(I, f^{s}\right) \neq 0$ for some positive integer $s$, then $I$ contains a periodic point, though the minimal period may be less than $s$. 
We are, of course, interested in more complicated dynamics. The simplest criterion is due to [2] where it is shown that if the spectral radius of $f_{N, 1}: H_{1}\left(N_{1} / N_{0},\left[N_{0}\right]\right) \rightarrow$ $H_{1}\left(N_{1} / N_{0},\left[N_{0}\right]\right)$ is greater than one, then the entropy of $\operatorname{Inv}(I, f)$ is positive. To obtain a more detailed description of the dynamics, we need to impose further restrictions on the index pair $\left(N_{1}, N_{0}\right)$. Following the ideas of $[23,22,21,20]$ assume that the sets $N_{i}$ are cubical and that

$$
\operatorname{cl}\left(N_{1} \backslash N_{0}\right)=\bigcup_{j=1}^{J} B_{j}
$$

where the $B_{j}$ are compact disjoint sets. Let $E_{j}:=\operatorname{cl}\left(N_{1} \backslash B_{j}\right)$. Then there is a continuous induced map $f_{B_{j}}:\left(N_{1} / E_{j},\left[E_{j}\right]\right) \rightarrow\left(N_{1} / E_{j},\left[E_{j}\right]\right)$. Observe that if $\pi_{j}:\left(N_{1} / N_{0},\left[N_{0}\right]\right) \rightarrow\left(N_{1} / E_{j},\left[E_{j}\right]\right)$ denotes the projection map, then $f_{B_{j}}=\pi_{j} \circ f_{N}$. Thus we can recover $f_{B_{j} *}$ from $f_{N *}$.

This information can be used to describe the invariant set in terms of symbolic dynamics as follows. Let $\Sigma_{J}=\{1, \ldots J\}^{\mathbb{N}}$ with the product topology. Define $\rho$ : $\operatorname{Inv}(I, f) \rightarrow \Sigma_{J}$ by

$$
(\rho(x))_{n}=j \quad \text { if } \quad f^{n}(x) \in B_{j} .
$$

Let $\gamma^{t+1}=\left(\gamma_{0}, \ldots, \gamma_{t}\right) \in\{1, \ldots J\}^{t+1}$. Define

$$
f_{\gamma^{t+1}}=f_{B_{\gamma_{t}} *} \circ \cdots \circ f_{B_{\gamma_{1}} *} .
$$

Let

$$
\Gamma:=\mathrm{cl}\left(\bigcup_{t \in \mathbb{N}}\left\{\gamma^{t+1} \in\{1, \ldots J\}^{t+1} \mid f_{\gamma^{t+1}}^{k} \neq 0 \forall k \in \mathbb{N}\right\}\right) \subset \Sigma_{J} .
$$

Then,

$$
\Gamma \subset \rho(\operatorname{Inv}(I, f)) .
$$

3. Reduction of $\Phi$. In this section we are going to describe in detail how to reduce the infinite dimensional map $\Phi$ to a combinatorial multivalued map $\mathcal{F}: \mathcal{G} \rightrightarrows \mathcal{G}$ on some finite set $\mathcal{G}$ which we can deal with on the computer. This reduction process involves three main steps as outlined in the Introduction. First we recast the map into a (single-valued) countable system $f$ of maps using a Galerkin projection. Second we define a finite-dimensional multivalued map $F$ in such a way that the dynamics of $f$ is captured by $F$ in a given subset of the phase space. Finally we discretize the phase space of $F$ using a finite cubical grid $\mathcal{G}$ and define a map $\mathcal{F}$ on $\mathcal{G}$ which captures the dynamics of $F$.

3.1. The Galerkin projection. As laid out in the Introduction, using Fourier modes $\varphi_{k}(x)=e^{i k x}, k \in \mathbb{Z}$, as the basis for $L^{2}$ we get the countable system of maps

$$
a_{k} \mapsto f_{k}\left(\left(a_{i}\right)_{i \geq 0}\right):=\mu b_{k}\left[a_{k}-\sum_{j+l+n=k} c_{j} a_{l} a_{n}\right], \quad k=0,1,2, \ldots,
$$

where $a_{k}=a_{-k}, b_{k}=b_{-k}, c_{k}=c_{-k} \in \mathbb{R}, k \in \mathbb{Z}$, are the coefficients of the Fourier expansions of $a, b$ and $c^{-1}$, respectively. 
3.2. Finite-dimensional approximation. We next need to reduce the countable system $f$ to a finite dimensional one. The idea is to do computations using the first $m$ coordinates of $f$ and to incorporate the neglected terms into a fixed error which makes the finite-dimensional map multivalued.

So we split the $f_{k}$ into a part which only depends on the variables $a_{0}, \ldots, a_{m-1}$ and the rest:

$$
f_{k}\left(\left(a_{i}\right)_{i \geq 0}\right)=f_{k}^{(m)}\left(a_{0}, \ldots, a_{m-1}\right)+f_{k}^{(m+)}\left(\left(a_{i}\right)_{i \geq 0}\right), \quad k=0,1,2, \ldots, .
$$

Now $f^{(m)}:=\left(f_{0}^{(m)}, \ldots, f_{m-1}^{(m)}\right)$ defines a map on $\mathbb{R}^{m}$. In order to deal with the error contributed by the $f_{k}^{(m+)}$ term we consider the dynamics of $f$ on the set

$$
Z=\prod_{k=0}^{\infty}\left[a_{k}^{-}, a_{k}^{+}\right]=W \times \prod_{k=m}^{\infty}\left[a_{k}^{-}, a_{k}^{+}\right]
$$

(where the $a_{k}^{ \pm}$are chosen in such a way that $Z$ is compact) and use interval arithmetic and the estimates in Section 5 to bound $f_{k}^{(m+)}(Z), k=0, \ldots, m-1$, by an interval $\varepsilon_{k}^{(m+)}[-1,1]$, i.e. we compute $\varepsilon_{k}^{(m+)}>0, k=0, \ldots, m-1$, such that

$$
f_{k}^{(m+)}(Z) \subset \varepsilon_{k}^{(m+)}[-1,1] .
$$

The finite-dimensional multivalued map $F^{(m)}: W \rightrightarrows \mathbb{R}^{m}$ is now defined as

$$
F^{(m)}\left(a_{0}, \ldots, a_{m-1}\right)=f^{(m)}\left(a_{0}, \ldots, a_{m-1}\right)+\prod_{k=0}^{m-1} \varepsilon_{k}^{(m+)}[-1,1] .
$$

Obtaining the bounds $a_{k}^{ \pm}$. One way to compute initial values for the bounds $a_{k}^{ \pm}$is to run a simulation of $f^{(L)}: \mathbb{R}^{L} \rightarrow \mathbb{R}^{L}$ for some large $L$. That is, for some arbitrary finite set $A \subset \mathbb{R}^{L}$ of points and some numbers $K_{0}<K_{1} \in \mathbb{N}$ we compute

$$
A_{K}:=\bigcup_{k=K_{0}}^{K_{1}}\left(f^{(L)}\right)^{k}(A) \text {. }
$$

For $k=0, \ldots, L-1$ set

$$
\begin{aligned}
& A_{k}^{-}:=\min \left\{a_{k} \mid\left(a_{0}, \ldots, a_{L-1}\right) \in A_{K}\right\} \\
& A_{k}^{+}:=\max \left\{a_{k} \mid\left(a_{0}, \ldots, a_{L-1}\right) \in A_{K}\right\} .
\end{aligned}
$$

Since the values of the bounds $a_{k}^{ \pm}$will have a strong impact on how large the errors $\varepsilon_{k}^{(m+)}$ will be, we decide to split these into two groups: we will use explicit bounds for $k<M$, where $M>m$ is some constant which will be determined by inspecting the values $A_{k}^{ \pm}$. For $k \geq M$ we will use a polynomial or an exponential decaying bound for $a_{k}^{ \pm}$, i.e. $a_{k}^{ \pm}= \pm \frac{A_{s}}{k^{s}}$ resp. $a_{k}^{ \pm}= \pm \frac{A_{s}}{s^{k}}, k \geq M$. The decay rate of the $A_{K}^{ \pm}$yields a first guess for the constants $A_{s}$ and $s$, i.e. we choose $A_{s}$ and $s$ such that

$$
\left[A_{k}^{-}, A_{k}^{+}\right] \subset \frac{A_{s}}{k^{s}}[-1,1], \quad \text { resp. } \quad\left[A_{k}^{-}, A_{k}^{+}\right] \subset \frac{A_{s}}{s^{k}}[-1,1]
$$

for $k=M, \ldots, L-1$. Now choose the $a_{k}^{ \pm}$such that

$$
\left[A_{k}^{-}, A_{k}^{+}\right] \subseteq\left[a_{k}^{-}, a_{k}^{+}\right] \text {for } k=0, \ldots, M-1,
$$


as well as

$$
a_{k}^{ \pm}:= \pm \frac{A_{s}}{k^{s}} \text { resp. } a_{k}^{ \pm}:= \pm \frac{A_{s}}{s^{k}} \quad \text { for } k \geq M
$$

Determining the projection dimension. We still have to determine the dimension $m$ used in the reduction process. If we choose $m$ too small, the errors $\varepsilon_{k}^{(m+)}$ will be too big and we will not be able to extract interesting dynamics on $F^{(m)}$. On the other hand, if $m$ is chosen too big, we will be doing unneccessary computations. So roughly speaking we will choose the smallest $m$, such that a numerical study of $F^{(m)}$ yields interesting results. In practice this essentially involves doing some sample computations using different values of $m$.

3.3. Finite representation. So far we deduced a finite-dimensional multivalued map $F^{(m)}$ from the original system $\Phi$. The next step will be to get a finite representation of $F^{(m)}$ which we can deal with in the computer. To this end we are partitioning the phase space $W$ of $F^{(m)}$ into a finite number of cubical sets. A cubical set is a subset $B$ of $\mathbb{R}^{m}$ of the form

$$
B=B(c, r)=\left\{x|| x_{k}-c_{k} \mid \leq r_{k}, k=0, \ldots, m-1\right\},
$$

where $c, r \in \mathbb{R}^{m}, r_{k} \geq 0$, are the center and radius of $B$. Note that the number of sets in a cubical partition of $W$ grows exponentially in the dimension $m$ of $W$, a fact that would render the following computational approach prohibitively expensive. For that reason we are actually not going to work with a partition of the whole of $W$ but instead with a cubical covering of the maximal invariant set of $F$ in $W$. This way the numerical effort is essentially determined by the dimension of the maximal invariant set - which typically is much smaller than $m$ (see [4]).

Note that $W$ is a cubical set. Let $c=\left(c_{0}, \ldots, c_{m-1}\right)$ be the center and $r=$ $\left(r_{0}, \ldots, r_{m-1}\right)$ be the radius of $W$, then by bisecting $W$ with respect to the $j$-th coordinate direction one obtains two cubical sets $B^{-}=B\left(c^{-}, \hat{r}\right)$ and $B^{+}=B\left(c^{+}, \hat{r}\right)$, where

$$
\hat{r}_{k}=\left\{\begin{array}{cl}
r_{k} & \text { for } k \neq j \\
r_{k} / 2 & \text { for } k=j
\end{array} \quad, \quad c_{k}^{ \pm}=\left\{\begin{array}{cl}
c_{k} & \text { for } k \neq j \\
c_{k} \pm r_{k} / 2 & \text { for } k=j
\end{array} .\right.\right.
$$

A cubical set which can be represented by iterating this subdivision process will be called a box. Note that a binary tree represents a certain set of boxes if one assigns a coordinate direction to each level (i.e. the set of all nodes with the same distance from the root) of the tree: the root corresponds to the box $W$ and all nodes of a given depth (i.e. on the same level) in the tree correspond to a subset of a cubical grid on $W$ (see [4] for a more detailed description of this approach). Denote by $\mathcal{B}_{k}$ the collection of all boxes represented by the nodes on depth $k$ of a tree (where the root has depth $0)$. For a subset $\mathcal{B} \subset \mathcal{B}_{k}$ let $|\mathcal{B}|$ denote the union of all boxes in $\mathcal{B}$. Let $o(\mathcal{B})$ be the set of all boxes in $\mathcal{B}_{k}$ which intersect $|\mathcal{B}|$, i.e. the smallest representable neighborhood of $|\mathcal{B}|$ in $\mathcal{B}_{k}$.

The multivalued map $F^{(m)}$ in a natural way defines a multivalued map $\mathcal{F}^{(m)}$ on $\mathcal{B}_{k}$ : For $B \in \mathcal{B}_{k}$ let $\mathcal{F}^{(m)}(B)$ be the set of all boxes in $\mathcal{B}_{k}$ which intersect the set $F^{(m)}(B)$. However, in order to allow for errors introduced when computing and representing $F^{(m)}(B)$, we will actually deal with an enclosure of $F^{(m)}$, i.e. a map $\mathcal{F}^{(m)}: \mathcal{B}_{k} \rightrightarrows \mathcal{B}_{k}$ such that

$$
F^{(m)}(B) \subset \operatorname{int}\left|\mathcal{F}^{(m)}(B)\right|
$$


for $B \in \mathcal{B}_{k}$. It is the map $\mathcal{F}^{(m)}$ that we are dealing with in the computer. In a concrete implementation it may be represented as e.g. a (sparse) matrix or a graph.

Computing an enclosure of $F$. Let us start by considering a single-valued map $g: \mathbb{R}^{m} \rightarrow \mathbb{R}^{m}$ (for example, consider the single-valued part $f^{(m)}$ of $F^{(m)}$ ). An approximate method to compute all boxes $B^{\prime}$ in the collection $\mathcal{B}_{k}$ which intersect the image $g(B)$ of a given box $B \in \mathcal{B}_{k}$ is to choose a finite set $T$ of test points in $B$ and to consider all boxes $B^{\prime}$ which contain at least one of the image points $f(T)$. This is the approach originally proposed in $[4,5]$. Note that due to the hierachical storage scheme of the boxes in a binary tree the computational complexity of determining the box $B^{\prime} \in \mathcal{B}_{k}$ which contains some specific image point is only $\mathcal{O}(k)$. In general this approach will not yield an enclosure of $g$. However, in [10] it has been shown how to extend this approach to compute an enclosure by constructing an appropriate mesh of test points in each box.

The approach used in this paper for the multivalued map is still different and based on the following observations:

1. For small enough boxes (i.e. large $k$ ) the image of a box $B \in \mathcal{B}_{k}$ under a map $g: \mathbb{R}^{m} \rightarrow \mathbb{R}^{m}$ is approximately given by its image under the linear part of $g$;

2. For a given cubical set $C \subset \mathbb{R}^{m}$ the set of all boxes $B^{\prime} \in \mathcal{B}_{k}$ which intersect $C$ can efficiently be determined by a single depth first search in the tree.

So the idea is to use the linear part of $f^{(m)}$ (the single-valued part of $F^{(m)}$ ) to compute an approximate image of a box $B$, to enclose this image by a cubical set and then to enlarge this cubical set by the errors made by neglecting the nonlinear terms of $f^{(m)}$, as well as the multivalued part of $F^{(m)}$. In doing these computations we use interval arithmetic as implemented in the BIAS, PROFIL and b4m libraries (see [13, 14, 25]) in order to control round-off errors.

Let us be more precise. Consider the box $B=B(c, r) \in \mathcal{B}_{k}$. For $h \in \mathbb{R}^{m}$ we can decompose $f^{(m)}$ as

$$
f^{(m)}(c+h)=f^{(m)}(c)+D f^{(m)}(c) h+f^{(m), n l}(c, h),
$$

where $f^{(m), n l}(c, 0)=0$. Compute $\varepsilon^{(m), n l}(c) \in \mathbb{R}^{m}$ such that

$$
\max _{h \in B(0, r)}\left|f^{(m), n l}(c, h)\right| \leq \varepsilon^{(m), n l}(c) .
$$

Then $F^{(m)}(B)$ will be contained in the cubical set $B\left(f^{(m)}(c), R\right)$, where

$$
R=\left|D f^{(m)}(c)\right| r+\varepsilon^{(m), n l}(c)+\varepsilon^{(m+)},
$$

and for a matrix $A=\left(a_{i j}\right) \in \mathbb{R}^{d, e}$ we write $|A|:=\left(\left|a_{i j}\right|\right) \in \mathbb{R}^{d, e}$. One should emphasize that the computation of $\varepsilon^{(m), n l}$ may eventually be expensive - it is not in our case. Now the enclosure $\mathcal{F}: \mathcal{B}_{k} \rightrightarrows \mathcal{B}_{k}$ of $F^{(m)}$ is defined in the following way: Let $\mathcal{F}(B)$ be the set of boxes which is intersected by the cubical set $B\left(f^{(m)}(c), R\right)$.

The following algorithm (when called as $\operatorname{cap}(\emptyset, W, C, k)$ ) computes the set $\mathcal{I}$ of all boxes in $\mathcal{B}_{k}$ which have a nonempty intersection with the cubical set $C$.

Algorithm 1.

$\mathcal{I}=\operatorname{cap}(\mathcal{I}, B, C, k)$

if $B \cap C \neq \emptyset$

if $\operatorname{depth}(B)=k$

$\mathcal{I}:=\mathcal{I} \cup\{B\}$

else

$\mathcal{I}:=\mathcal{I} \cup \operatorname{cap}\left(\mathcal{I}, B^{+}, C, k\right) \cup \operatorname{cap}\left(\mathcal{I}, B^{-}, C, k\right)$

return $\mathcal{I}$ 
Here the function $\operatorname{depth}(B)$ returns $k$ if $B \in \mathcal{B}_{k}$, and $B^{+}$and $B^{-}$are the two boxes which result from bisecting $B$ with respect to some coordinate direction - as defined by the tree used to store the box collections.

4. Computing isolating neighborhoods. After reducing the map $\Phi$ to a combinatorial map $\mathcal{F}$ on a grid we are now interested in computing isolating neighborhoods for $\mathcal{F}$ which isolate certain sets of interest. In particular we will be interested in isolating periodic points, connecting orbits and - combining these two - invariant sets with complicated dynamics. These neighborhoods will translate directly into isolating neighborhoods for the multivalued map $F$ (see Section 1) and - together with certain conditions on $f$ - into isolating neighborhoods for $f$ and thus for $\Phi$ (see Section 2).

Szymczak ([22]) describes an algorithm for finding isolating neighborhoods of $\mathcal{F}$ in a given subset $\mathcal{B}$ of $\mathcal{B}_{k}$. The basic idea is to "cut" the proper pieces out of $\mathcal{B}$ until the resulting collection satisfies the criterion (1.12). This method has the drawback that one has to choose a suitable set $\mathcal{B}$ apriori and may eventually end up with the empty set as a trivial isolating neighborhood. The approach we will describe now proceeds in some sense in the opposite direction: one starts with a guess for an isolating neighborhood of some interesting invariant set and "fattens" this set by adding neighborhoods until the condition (1.12) is satisfied.

4.1. Guessing isolating neighborhoods. Guesses for isolating neighborhoods of specific invariant sets of $\mathcal{F}$ can easily be obtained:

- $k$-periodic points of $\mathcal{F}$ are identified by nonzero diagonal entries of $M_{\mathcal{F}}^{k}$, where the transition matrix $M_{\mathcal{F}}=\left(m_{i j}\right)$ is given by

$$
m_{i j}= \begin{cases}1, & \text { if } B_{j} \in \mathcal{F}\left(B_{i}\right) \\ 0, & \text { else }\end{cases}
$$

and $\mathcal{B}_{k}=\left\{B_{1}, \ldots, B_{p}\right\}$

- more generally, recurrent sets of $\mathcal{F}$ are given by strongly connected components of a graph representing $\mathcal{F}$;

- in this graph connecting orbits of $\mathcal{F}$ can be identified by shortest path algorithms (like e.g. the Dijkstra-algorithm, see [6]);

- Szymzcak [22] describes how to compute the maximal invariant set of $\mathcal{F}$.

4.2. Turning the guess into a true isolating neighborhood. Once a guess $\tilde{\mathcal{I}}$ for an isolating neighborhood of $\mathcal{F}$ has been computed, we construct a (true) isolating neighborhood $\mathcal{I}$ containing $\tilde{\mathcal{I}}$ by the following procedure.

Algorithm 2.

$\mathcal{I}=\operatorname{make} \_$isolated $(\tilde{\mathcal{I}})$

$\mathcal{I}:=\operatorname{Inv}(\tilde{\mathcal{I}}, \mathcal{F})$

while $o(\mathcal{I}) \not \subset \tilde{\mathcal{I}}$

$\tilde{\mathcal{I}}:=\tilde{\mathcal{I}} \cup o(\mathcal{I})$

$\mathcal{I}:=\operatorname{Inv}(\tilde{\mathcal{I}}, \mathcal{F})$

if $\mathcal{I} \subset \operatorname{int}|o(\mathcal{I})|$ return $\mathcal{I}$

else return $\emptyset$

By construction this algorithm returns a combinatorial isolating neighborhood $\mathcal{I}$ for $\mathcal{F}$. Similar to the procedure proposed in [22] one may end up with the empty set, in which case the set $|\mathcal{I}|$ touched the boundary of $W$.

4.3. Tightening the isolating neighborhood. So far we computed an isolating neighborhood $\mathcal{I} \subset \mathcal{B}_{k}$ for $\mathcal{F}$. We are now going to address the question of how to 
improve this neighborhood in the sense that one gets a tighter covering of the underlying invariant set. This process of tightening involves three steps which repeatedly may be applied until the desired accurracy has been reached or the machine resources are exhausted.

4.3.1. The subdivision algorithm. In [4] Dellnitz and Hohmann describe a subdivision procedure for the computation of relative global attractors of maps. The basic idea of the therein advocated multilevel approach is to iteratively refine a given collection of boxes and then to select a certain subset of the refined collection which contains the dynamics of interest.

Algorithm 3 ([4]). Given the initial collection $\mathcal{B}_{0}$, one inductively obtains $\mathcal{B}_{k}$ from $\mathcal{B}_{k-1}$ for $k=1,2, \ldots$ in two steps.

1. Subdivision: Construct a new collection $\hat{\mathcal{B}}_{k}$ by bisecting each box in $\mathcal{B}_{k-1}$ with respect to some coordinate direction.

2. Selection: Compute the relevant subset $\mathcal{B}_{k}$ of $\hat{\mathcal{B}}_{k}$.

The second step obviously defines which sets will be contained in $\mathcal{B}_{k}$. In our case, since we want to compute isolating neighborhoods, we are interested in covering the maximal invariant set. So we set

$$
\mathcal{B}_{k}=\operatorname{Inv}\left(\hat{\mathcal{B}}_{k}, \mathcal{F}\right)
$$

(cf. [22]), where we start with $\mathcal{B}_{0}=\mathcal{I}$. The following statement formalizes that we do not loose the isolation property for the tightened neighborhood. Its proof is essentially the same as for theorem 2.2 in [22].

Proposition 4.1. Let $\mathcal{B}_{0}=\mathcal{I}$ be a collection of boxes which is an isolating neighborhood for $\mathcal{F}: \mathcal{B}_{0} \rightrightarrows \mathcal{B}_{0}$. Then the collections $\mathcal{B}_{k}, k=1,2, \ldots$, computed by Algorithm 3 with selection criterion (4.1), are also isolating neighborhoods for $\mathcal{F}: \mathcal{B}_{k} \rightrightarrows \mathcal{B}_{k}$.

In practice it will not be advisable to perform more than a few steps of the subdivision procedure at once. Recall that the combinatorial map $\mathcal{F}$ has been defined on basis of the multivalued map $F=F^{(m)}$ which incorporated the errors $\varepsilon_{k}^{(m+)}$ contributed to the dynamics of $f^{(m)}$ by the higher order modes. As soon as these errors and the size of the boxes in the current collection $\mathcal{B}_{k}$ are on the same order of magnitude a further refinement using Algorithm 3 does not make sense any more. Instead one will first have to make the $\varepsilon_{k}^{(m+)}$ smaller by the following two methods.

4.3.2. Updating the bounds $a_{k}^{ \pm}$. Recall that we start all computations on the infinite-dimensional cube

$$
Z=W \times V=\prod_{k=0}^{\infty}\left[a_{k}^{-}, a_{k}^{+}\right]
$$

where we obtained initial guesses for the $a_{k}^{ \pm}$by running a simulation of $f^{(m)}$ for a large $m$. The size of the $a_{k}^{ \pm}$will have a crucial influence on the size of the errors $\varepsilon_{k}^{(m)+}$ introduced in the multivalued map $F^{(m)}$ by estimating the contribution from the neglected modes. Since these errors determine the precision of the computations (and whether we will be able to do an interesting computation in the first place) we are interested in making $\left|a_{k}^{ \pm}\right|$as small as possible. Remember that we splitted the variables $a_{k}$ into three groups:

1. $0 \leq k<m$ : these are the actual variables we are computing with. We are getting tight bounds on these by encapsulating our covering $\mathcal{B}_{\ell}$ of the maximal 
invariant set of $F^{(m)}: W \rightrightarrows \mathbb{R}^{m}$ into a cube, i.e. we choose $a_{k}^{ \pm}, 0 \leq k<m$, such that

$$
\left|\mathcal{B}_{\ell}\right| \subset \prod_{k=0}^{m-1}\left[a_{k}^{-}, a_{k}^{+}\right] ;
$$

2. $m \leq k<M$ : for these we store bounds on the $a_{k}$ explicitly. As laid out in Section 2 in order to lift the index information to the full system it is sufficient to require that the map $f$ satisfies

$$
f_{k}(Z) \subset\left(a_{k}^{-}, a_{k}^{+}\right), \quad k \geq m .
$$

Note that via the estimates in Section 5 we are able to bound $f_{k}(Z)$ in terms of an interval. What is more, since we are dealing with a polynomial nonlinearity, whenever we decrease $\left|a_{\ell}^{ \pm}\right|$for some $\ell$, the bound on $f_{k}(Z)$ will also decrease. This is the basis for the following update scheme for the $a_{k}^{ \pm}$ with $m \leq k<M$ : For $k=m, \ldots, M-1$ we compute the new $\left[a_{k}^{-}, a_{k}^{+}\right]$as the interval bounding $f_{k}(Z)$ using the estimates in Section 5 , in particular Corollary 5.5 resp. 5.11 .

3. $k \geq M$ : these variables are bounded by a decay law of exponential or polynomial type, i.e. $a_{k}^{ \pm}= \pm \frac{A_{s}}{s^{k}}$ resp. $a_{k}^{ \pm}= \pm \frac{A_{s}}{k^{s}}$. In this case one gets tighter bounds by updating the constants $s$ and $A_{s}$. This is detailed in Section 5 (Lemma 5.7 and Lemma 5.13).

4.3.3. Increasing the projection dimension $m$. The third method to get tighter bounds on the computed invariant set is to increase the number of Galerkin modes used in the computations. We write $\mathcal{B}_{k}=\mathcal{B}_{k}^{(m)}$ for a box collection in $\mathbb{R}^{m}$. Define

$$
\mathcal{B}_{k}^{(m+1)}=\left\{B \times\left[a_{m}^{-}, a_{m}^{+}\right]: B \in \mathcal{B}_{k}^{(m)}\right\}
$$

and let $\mathcal{F}^{(m+1)}: \mathcal{B}_{k}^{(m+1)} \rightrightarrows \mathcal{B}_{k}^{(m+1)}$ be the combinatorial map defined by the multivalued map $F^{(m+1)}: W^{(m+1)} \rightrightarrows \mathbb{R}^{m+1}, W^{(m+1)}=W^{(m)} \times\left[a_{m}^{-}, a_{m}^{+}\right]$. Now let $\mathcal{I}^{(m)} \subset \mathcal{B}_{k}^{(m)}$ be an isolating neighborhood for $\mathcal{F}^{(m)}: \mathcal{B}_{k}^{(m)} \rightrightarrows \mathcal{B}_{k}^{(m)}$ and set

$$
\mathcal{I}^{(m+1)}=\left\{B \times\left[a_{m}^{-}, a_{m}^{+}\right]: B \in \mathcal{I}^{(m)}\right\} .
$$

Using Corollary 2.4 we get that $\mathcal{I}^{(m+1)}$ is an isolating neighborhood for $\mathcal{F}^{(m+1)}$ and in particular, the index information of a corresponding index pair carries over from dimension $m$ to $m+1$.

5. Error Bounds (Polynomial Nonlinearity). As discussed in Section 1, we need to study maps of the form:

$$
\left(a_{k}\right)_{k} \mapsto b_{k} \sum_{n_{0}, \ldots, n_{p-1} \in \mathbb{Z}} c_{n_{0}}^{p} a_{n_{1}} \ldots a_{n_{p-1}} a_{k-\left(n_{0}+\ldots+n_{p-1}\right)}
$$

corresponding to a monomial $c_{p} a^{p}$ of the growth function, where $a_{k}=a_{-k}, b_{k}$ is the $k$-th eigenvalue for the underlying linear operator, and $c_{k}^{p}$ and $a_{k}$ are the $k$-th coefficients of the expansions of $c_{p}$, and $a$ respectively. The maps for a polynomial growth function are the sums of the maps for the monomials. It is important to note, 
however, that when written in terms of an appropriate basis, a wide variety of systems with polynomial nonlinearities produce maps of this form.

We next restrict the domain to a set $Z=\prod_{k}\left[a_{k}^{-}, a_{k}^{+}\right]=W \times \prod_{k \geq M}\left[a_{k}^{-}, a_{k}^{+}\right]$where for $k \geq M, 0 \in\left[a_{k}^{-}, a_{k}^{+}\right]$and $a_{k}^{+}-a_{k}^{-}$satisfies some decay rule. The justification of this restriction is given by the following lemma.

Lemma 5.1. Any invariant set of $\Phi$ is contained in a set $Z$ of the form given above, where the decay in the higher modes reflects the decay of the eigenvalues $b_{k}$ of the linear operator.

Proof. Let $a \in X$ with corresponding Fourier expansion $\sum_{k} a_{k} \varphi_{k}$.

The projection of the image of $a$ onto the $k$-th mode is,

$$
\begin{aligned}
\left\langle\Phi(a), \varphi_{k}\right\rangle & =\int_{-\pi}^{\pi} \Phi(a)(y) \varphi_{k}(y) d y \\
& =\int_{-\pi}^{\pi} \frac{1}{2 \pi} \int_{-\pi}^{\pi} b(x, y) g(a)(x) \varphi_{k}(y) d x d y \\
& =\frac{1}{2 \pi} \sum_{n} b_{n} \int_{-\pi}^{\pi} \varphi_{n}(x) g[a](x)\left(\int_{-\pi}^{\pi} \varphi_{n}(-y) \varphi_{k}(y) d y\right) d x \\
& =b_{k} \int_{-\pi}^{\pi} \varphi_{k}(x) g[a](x) d x \\
& =b_{k}\left\langle g[a], \varphi_{k}\right\rangle \\
& \leq C_{g, a} b_{k}
\end{aligned}
$$

for some constant $C_{g, a}$ that does not depend on $k$.

In particular, any set which is invariant (forward and backward in time) must be contained in a set of the form of $Z=\prod_{k}\left[a_{k}^{-}, a_{k}^{+}\right]$, where for $k$ sufficiently large $(k \geq M), a_{k}^{+}-a_{k}^{-}$is shrinking according to the contraction given by the eigenvalue $b_{k}$.

In practice, the domain intervals are determined by preliminary simulations, with a decay rule reflecting the decay found in the eigenvalues for the linear operator. We now use interval arithmetic to determine bounds for the error term $f^{(m+)}(a)$ resulting from considering only the first $m$ modes as variables. To emphasize our use of intervals we will write $\tilde{a}_{k}:=\left[a_{k}^{-}, a_{k}^{+}\right]$.

5.1. Exponential Decay. In this section, we assume that the eigenvalues $b_{k}$ of the linear operator decay exponentially. That is, there exist constants $b>1$ and $B>0$ such that $\left|b_{k}\right| \leq \frac{B}{b^{k}}$. In this case, we begin by setting $\tilde{a}_{k}=\left[-\frac{A_{s}}{s^{k}}, \frac{A_{s}}{s^{k}}\right]$ for all $k \geq M$ where the constants $A_{s}>0$ and $s>1$ are determined by preliminary simulations. In order to reduce the number of cases we need to consider, we will extend the asymptotic bounds to all $k$. In other words, $\tilde{a}_{k} \subseteq \frac{A}{s^{|k|}}[-1,1]$ for all $k \in \mathbf{Z}$, where $A=\max \left\{A_{s}, \max _{0 \leq k<M, a_{k} \in \tilde{a}_{k}} s^{k}\left|a_{k}\right|\right\}$. For future computations, fix $\beta$ such that $\frac{b}{s}<\beta<b$.

Consider the monomial $a^{p}$ of order $p$ with coefficient 1 . We begin by bounding the sum in the corresponding maps.

LEMMA 5.2. For all $k \in \mathbb{Z}$,

$$
\sum_{n_{1}, \ldots, n_{p-1} \in \mathbb{Z}} \tilde{a}_{n_{1}} \ldots \tilde{a}_{n_{p-1}} \tilde{a}_{k-\left(n_{1}+\ldots+n_{p-1}\right)} \subseteq \frac{\alpha^{p-1} A^{p}}{s^{|k|}}\left(\frac{b}{\beta}\right)^{|k|}[-1,1]
$$


where $\alpha=\left(\frac{2}{\ln s}+\frac{b}{\beta \ln (b / \beta)}\right)$.

Proof. This formula holds for $p=1$, since

$$
\tilde{a}_{k} \subseteq \frac{A}{s^{|k|}}[-1,1] \subseteq \frac{A}{s^{|k|}}\left(\frac{b}{\beta}\right)^{|k|}[-1,1]
$$

Assume the formula holds for $p-1$. Then, for $k \geq 0$,

$$
\begin{aligned}
& \sum_{n_{1}, \ldots, n_{p-1}} \tilde{a}_{n_{1}} \cdots \tilde{a}_{n_{p-1}} \tilde{a}_{k-\left(n_{1}+\ldots+n_{p-1}\right)} \\
& =\sum_{n_{1}} \tilde{a}_{n_{1}} \sum_{n_{2}, \ldots, n_{p-1}} \tilde{a}_{n_{2}} \cdots \tilde{a}_{n_{p-1}} \tilde{a}_{\left(k-n_{1}\right)-\left(n_{2}+\ldots+n_{p-1}\right)} \\
& \subseteq \sum_{n_{1}} \tilde{a}_{n_{1}} \frac{\alpha^{p-2} A^{p-1}}{s^{\left|k-n_{1}\right|}}\left(\frac{b}{\beta}\right)^{\left|k-n_{1}\right|}[-1,1] \\
& \subseteq\left(\sum_{n_{1} \leq k} \tilde{a}_{n_{1}} \frac{\alpha^{p-2} A^{p-1}}{s^{k-n_{1}}}\left(\frac{b}{\beta}\right)^{k-n_{1}}+\sum_{n_{1}>k} \tilde{a}_{n_{1}} \frac{\alpha^{p-2} A^{p-1}}{s^{n_{1}-k}}\left(\frac{b}{\beta}\right)^{n_{1}-k}\right)[-1,1] \\
& \subseteq\left(\sum_{n_{1}>0} \frac{A \alpha^{p-2} A^{p-1}}{s^{k+2 n_{1}}}\left(\frac{b}{\beta}\right)^{k+n_{1}}+\sum_{n_{1}=0}^{k} \frac{A \alpha^{p-2} A^{p-1}}{s^{k}}\left(\frac{b}{\beta}\right)^{k-n_{1}}\right. \\
& \left.+\sum_{n_{1}>k} \frac{A \alpha^{p-2} A^{p-1}}{s^{2 n_{1}-k}}\left(\frac{b}{\beta}\right)^{n_{1}-k}\right)[-1,1] \\
& \subseteq\left(\frac{\alpha^{p-2} A^{p}}{s^{k}}\left(\frac{b}{\beta}\right)^{k}\left(\frac{b}{s \beta}\right)^{0} \sum_{n_{1}>0} \frac{1}{s^{n_{1}}}+\frac{\alpha^{p-2} A^{p}}{s^{k}}\left(\frac{b}{\beta}\right)^{k} \sum_{n_{1}=0}^{k}\left(\frac{\beta}{b}\right)^{n_{1}}\right. \\
& \left.+\alpha^{p-2} A^{p} s^{k}\left(\frac{\beta}{b}\right)^{k}\left(\frac{b}{s \beta}\right)^{k} \sum_{n_{1}>k} \frac{1}{s^{n_{1}}}\right)[-1,1] \\
& \subseteq\left(\frac{\alpha^{p-2} A^{p}}{s^{k}}\left(\frac{b}{\beta}\right)^{k} \int_{0}^{\infty} s^{-x} d x+\frac{\alpha^{p-2} A^{p}}{s^{k}}\left(\frac{b}{\beta}\right)^{k} \int_{-1}^{\infty}\left(\frac{\beta}{b}\right)^{x} d x\right. \\
& \left.+\alpha^{p-2} A^{p} \int_{k}^{\infty} s^{-x} d x\right)[-1,1] \\
& \subseteq \frac{\alpha^{p-2} A^{p}}{s^{k}}\left(\frac{b}{\beta}\right)^{k}\left(\frac{2}{\ln s}+\frac{b}{\beta \ln (b / \beta)}\right)[-1,1] \\
& =\frac{\alpha^{p-1} A^{p}}{s^{k}}\left(\frac{b}{\beta}\right)^{k}[-1,1]
\end{aligned}
$$

The case $k<0$ may be reduced to the previous case via change of indices.

$\square$

If the expansion of the coefficient function exhibits similar decay to that of $\tilde{a}_{k}$, then we may extend this argument to the maps corresponding to $c a^{p}$.

Corollary 5.3. If there exists a constant $C$ such that $c_{n} \in \tilde{c}_{n}:=\frac{C}{s^{|n|}}[-1,1]$ for all $n$, then 


$$
\sum_{n_{0}, n_{1}, \ldots, n_{p-1} \in \mathbf{Z}} \tilde{c}_{n_{0}} \tilde{a}_{n_{1}} \cdots \tilde{a}_{n_{p-1}} \tilde{a}_{k-\left(n_{0}+\ldots+n_{p-1}\right)} \subseteq \frac{\alpha^{p} A^{p} C}{s^{k}}\left(\frac{b}{\beta}\right)^{k}[-1,1]
$$

We now take advantage of the explicit bounds $\tilde{a}_{n}, 0 \leq n<\bar{M}$, instead of using only the extended asymptotic bounds $\frac{A_{s}}{s^{n}}[-1,1]$. By increasing $\bar{M}$, the error computations become more costly while giving tighter bounds on error terms.

Lemma 5.4. For $0 \leq k<\bar{M}$,

$$
\begin{aligned}
\sum_{n_{1}, \ldots, n_{p-1} \in \mathbb{Z}} \tilde{a}_{n_{1}} \cdots \tilde{a}_{n_{p-1}} \tilde{a}_{k-\left(n_{1}+\ldots+n_{p-1}\right)} & \\
& \subseteq \sum_{\left|n_{1}\right|, \ldots,\left|n_{p-1}\right| \leq \bar{M}} \tilde{a}_{n_{1}} \cdots \tilde{a}_{n_{p-1}} \tilde{a}_{k-\left(n_{1}+\ldots+n_{p-1}\right)} \\
& \quad+\frac{(p-1) \alpha^{p-2} A^{p-1} A_{s}}{s^{\bar{M}} \ln s}\left[\left(\frac{b}{s \beta}\right)^{\bar{M}-k}+\left(\frac{b}{s \beta}\right)^{\bar{M}+k}\right][-1,1]
\end{aligned}
$$

for any $\bar{M}>0$.

Proof.

$$
\begin{aligned}
\sum_{n_{1}, \ldots, n_{p-1} \in \mathbb{Z}} \tilde{a}_{n_{1}} \cdots \tilde{a}_{n_{p-1}} \tilde{a}_{k-\left(n_{1}+\ldots+n_{p-1}\right)} & \\
& \subseteq \sum_{\left|n_{1}\right|, \ldots,\left|n_{p-1}\right| \leq M^{*}} \tilde{a}_{n_{1}} \cdots \tilde{a}_{n_{p-1}} \tilde{a}_{k-\left(n_{1}+\ldots+n_{p-1}\right)} \\
& \quad+\sum_{i=1}^{p-1} \max _{a_{n} \in \tilde{a}_{n}}\left\{\sum_{n_{j} \in \mathbb{Z},\left|n_{i}\right|>M}\left|a_{n_{1}}\right| \cdots\left|a_{n_{p-1}}\right|\left|a_{k-\left(n_{1}+\ldots+n_{p-1}\right)}\right|\right\}[-1,1]
\end{aligned}
$$

By symmetry,

$$
\sum_{n_{j} \in \mathbb{Z},\left|n_{i}\right|>\bar{M}}\left|a_{n_{1}}\right| \cdots\left|a_{n_{p-1}}\right|\left|a_{k-\left(n_{1}+\ldots+n_{p-1}\right)}\right|=\sum_{n_{j} \in \mathbb{Z},\left|n_{1}\right|>\bar{M}}\left|a_{n_{1}}\right| \cdots\left|a_{n_{p-1}}\right|\left|a_{k-\left(n_{1}+\ldots+n_{p-1}\right)}\right|
$$

for $i=1, \ldots, p-1$.

Using the previous asymptotic estimates,

$$
\begin{aligned}
\sum_{n_{j} \in \mathbb{Z},\left|n_{1}\right|>\bar{M}}\left|a_{n_{1}}\right| & \cdots\left|a_{n_{p-1}}\right|\left|a_{k-\left(n_{1}+\ldots+n_{p-1}\right)}\right| \\
\leq & \sum_{n_{1}>\bar{M}} \frac{A_{s}}{s^{n_{1}}} \frac{\alpha^{p-2} A^{p-1}}{s^{n_{1}-k}}\left(\frac{b}{\beta}\right)^{n_{1}-k} \\
& \quad+\sum_{n_{1}<-\bar{M}} \frac{A_{s}}{s^{-n_{1}}} \frac{\alpha^{p-2} A^{p-1}}{s^{k-n_{1}}}\left(\frac{b}{\beta}\right)^{k-n_{1}} \\
= & \alpha^{p-2} A^{p-1} A_{s} s^{k}\left(\frac{\beta}{b}\right)^{k}\left(\frac{b}{s \beta}\right)^{\bar{M}} \sum_{n_{1}>\bar{M}} \frac{1}{s^{n_{1}}}
\end{aligned}
$$




$$
\begin{gathered}
+\sum_{n_{1}>\bar{M}} \frac{A_{s}}{s^{n_{1}}} \frac{\alpha^{p-2} A^{p-1}}{s^{k+n_{1}}}\left(\frac{b}{\beta}\right)^{k+n_{1}} \\
\leq \alpha^{p-2} A^{p-1} A_{s} s^{k}\left(\frac{\beta}{b}\right)^{k}\left(\frac{b}{s \beta}\right)^{\bar{M}} \int_{\bar{M}}^{\infty} s^{-x} d x \\
\quad+\frac{\alpha^{p-2} A^{p-1} A_{s}}{s^{k}}\left(\frac{b}{\beta}\right)^{k}\left(\frac{b}{s \beta}\right)^{\bar{M}} \int_{\bar{M}}^{\infty} s^{-x} d x \\
=\frac{\alpha^{p-2} A^{p-1} A_{s}}{s^{\bar{M}} \ln s}\left[\left(\frac{b}{s \beta}\right)^{\bar{M}-k}+\left(\frac{b}{s \beta}\right)^{\bar{M}+k}\right]
\end{gathered}
$$

Therefore,

$$
\begin{aligned}
\sum_{n_{1}, \ldots, n_{p-1} \in \mathbb{Z}} \tilde{a}_{n_{1}} \cdots \tilde{a}_{n_{p-1}} \tilde{a}_{k-\left(n_{1}+\ldots+n_{p-1}\right)} & \subseteq \sum_{\left|n_{1}\right|, \ldots,\left|n_{p-1}\right| \leq \bar{M}} \tilde{a}_{n_{1}} \cdots \tilde{a}_{n_{p-1}} \tilde{a}_{k-\left(n_{1}+\ldots+n_{p-1}\right)} \\
& \quad+\frac{(p-1) \alpha^{p-2} A^{p-1} A_{s}}{s^{\bar{M}} \ln s}\left[\left(\frac{b}{s \beta}\right)^{\bar{M}-k}+\left(\frac{b}{s \beta}\right)^{\bar{M}+k}\right][-1,1]
\end{aligned}
$$

$\square$

By a similar argument, we obtain the following corollary.

Corollary 5.5. For $0 \leq k<\bar{M}$,

$$
\begin{aligned}
\sum_{n_{0}, \ldots, n_{p-1} \in \mathbb{Z}} \tilde{c}_{n_{0}}^{p} \tilde{a}_{n_{1}} \cdots & \tilde{a}_{n_{p-1}} \tilde{a}_{k-\left(n_{0}+\ldots+n_{p-1}\right)} \\
\subseteq & \sum_{\left|n_{0}\right|, \ldots,\left|n_{p-1}\right| \leq \bar{M}} \tilde{c}_{n_{0}}^{p} \tilde{a}_{n_{1}} \cdots \tilde{a}_{n_{p-1}} \tilde{a}_{k-\left(n_{0}+\ldots+n_{p-1}\right)} \\
& +\frac{\alpha^{p-1} A^{p} C}{s^{\bar{M}} \ln s}\left[\left(\frac{b}{s \beta}\right)^{\bar{M}-k}+\left(\frac{b}{s \beta}\right)^{\bar{M}+k}\right][-1,1] \\
& +\frac{(p-1) \alpha^{p-1} A^{p-1} C A_{s}}{s^{\bar{M}} \ln s}\left[\left(\frac{b}{s \beta}\right)^{\bar{M}-k}+\left(\frac{b}{s \beta}\right)^{\bar{M}+k}\right][-1,1]
\end{aligned}
$$

for any $\bar{M}>0$.

Notice that the sum in these last estimates is finite. Therefore, we may decide on a case-by-case basis which of these terms contain only variables $\left(a_{k}\right.$ with $\left.0 \leq k<m\right)$. These terms are included in the finite-dimensional map, $f^{(m)}$, and should not be considered when bounding the error term, $f^{(m+)}$. In the remaining terms, we may use the explicit interval bounds instead of the extended asymptotic bounds. This, in principle, should give us a tighter bound on the error.

Corollary 5.6. For $0 \leq k<m$ and $\bar{M}>0$, the error in the $k$ th coordinate map, $\left[f^{(m+)}(Z)\right]_{k}$, corresponding to a nonlinear term of the form $c_{p} a^{p}$ is bounded by

$$
\begin{aligned}
{\left[f^{(m+)}(Z)\right]_{k} \subseteq } & \sum_{\left|n_{0}\right|, \ldots,\left|n_{p-1}\right| \leq \bar{M}} \overline{c_{n_{0}}^{p} a_{n_{1}} \cdots a_{n_{p-1}} a_{k-\left(n_{0}+\ldots+n_{p-1}\right)}} \\
& +\left(A+(p-1) A_{s}\right) \frac{(\alpha A)^{p-1} C}{s^{\bar{M}} \ln s}\left[\left(\frac{b}{s \beta}\right)^{\bar{M}-k}+\left(\frac{b}{s \beta}\right)^{\bar{M}+k}\right][-1,1]
\end{aligned}
$$


where $\overline{c_{n_{0}}^{p} a_{n_{1}} \cdots a_{n_{p-1}} a_{k-\left(n_{0}+\ldots+n_{p-1}\right)}}$ is 0 if all of the indices have absolute value less than $m$ and is the interval product $\tilde{c}_{n_{0}}^{p} \tilde{a}_{n_{1}} \cdots \tilde{a}_{n_{p-1}} \tilde{a}_{k-\left(n_{0}+\ldots+n_{p-1}\right)}$ otherwise.

Besides computing the errors for the multivalued map, we also wish to update both the explicit interval bounds $(0 \leq k<M)$ and the asymptotic bounds for the tail of the sequence $k \geq M$. For the first set of updates, $(0 \leq k<M)$, we may use the estimates given in Corollary 5.5. When updating the bounds for $k \geq M$, we use the computations given in Corollary 5.3 as follows.

LEMmA 5.7. Suppose the nonlinearity is $g(a)=\sum_{p=0}^{d} c_{p} a^{p}$ with the expansions of the coefficient functions satisfying the decay rules $c_{n}^{p} \in \tilde{c}_{n}^{p}:=\frac{C_{p}}{s^{|n|}}[-1,1]$ for some constants $C_{p}$. Then for $k \geq M$, we may set new bounds $\tilde{a}_{k}$ to be $\frac{A_{\beta s}}{(\beta s)^{k}}[-1,1]$ where

$$
A_{\beta s}=B\left(C_{0}+\alpha A C_{1}+\ldots+\alpha^{d} A^{d} C_{d}\right)
$$

Proof.

For a fixed $k \geq M$, we have bounds for the image of the corresponding map using the bounds for $\left|b_{k}\right|$ and the bounds for the sum given by Corollary 5.3.

The projection of the image of $Z$ under the full map onto the $k$ th mode is

$$
\begin{aligned}
{[F(Z)]_{k} } & =b_{k} \sum_{p=0}^{d} \sum_{n_{0}, \ldots n_{p-1}} \tilde{c}_{n_{0}}^{p} \tilde{a}_{n_{1}} \ldots \tilde{a}_{k-\left(n_{0}+\ldots+n_{p-1}\right)} \\
& \subseteq \frac{B}{b^{k}} \frac{1}{s^{k}}\left(\frac{b}{\beta}\right)^{k}\left(C_{0}+\alpha A C_{1}+\ldots+\alpha^{d} A^{d} C_{d}\right)[-1,1] \\
& =\frac{A_{\beta s}}{(\beta s)^{k}}[-1,1]
\end{aligned}
$$

where $A_{\beta s}$ is as given in the statement of the lemma.

By setting the new bounds $\tilde{a}_{k}$ to be the bounds on the image $[F(Z)]_{k}$, we preserve isolation.

5.2. Polynomial Decay. We now consider the case when the eigenvalues, $b_{k}$, for the linear operator exhibit polynomial decay. In other words, there exist constants $b>1$ and $B>0$ such that $\left|b_{k}\right| \leq \frac{B}{|k|^{b}}$ for all $k \in \mathbf{Z} \backslash\{\mathbf{0}\}$. Again, we assume that the sequence exhibits similar decay to that of the eigenvalues. That is, for some constants $A_{s}>0$ and $s>1$ initially given by simulations, $\tilde{a}_{k}=\frac{A_{s}}{|k|^{s}}[-1,1]$ for all $k>M$. Set $A=\max \left\{A_{s}, \max _{a_{0} \in \tilde{a}_{0}}\left|a_{0}\right|, \max _{0<k<M, a_{k} \in \tilde{a}_{k}}|k|^{s}\left|a_{k}\right|\right\}$. Then $\tilde{a}_{k} \subseteq \frac{A}{|k|^{s}}[-1,1]$ for all $k \in \mathbf{Z} \backslash\{\mathbf{0}\}$ and $\tilde{a}_{0} \subseteq A[-1,1]$. The following estimates are similar to those given in the exponential decay case.

Lemma 5.8. Let $\alpha=\frac{2}{s-1}+2+3.5 \cdot 2^{s}$. Then

$$
\sum_{n_{1}, \ldots, n_{p-1} \in \mathbb{Z}} \tilde{a}_{n_{1}} \cdots \tilde{a}_{n_{p-1}} \tilde{a}_{k-\left(n_{1}+\ldots+n_{p-1}\right)} \subseteq\left\{\begin{array}{cc}
\frac{\alpha^{p-1} A^{p}}{|k|^{s}}[-1,1] & k \neq 0 \\
\alpha^{p-1} A^{p}[-1,1] & k=0
\end{array}\right.
$$

Proof. For $p=1$, this inequality holds for all $k$. Now assume that

$$
\sum_{n_{1}, \ldots, n_{p-2} \in \mathbb{Z}} \tilde{a}_{n_{1}} \cdots \tilde{a}_{n_{p-2}} \tilde{a}_{k-\left(n_{1}+\ldots+n_{p-2}\right)} \subseteq \begin{cases}\frac{\alpha^{p-2} A^{p-1}}{\mid k^{s}}[-1,1] & k \neq 0 \\ \alpha^{p-2} A^{p-1}[-1,1] & k=0\end{cases}
$$


For $k=0$,

$$
\begin{aligned}
\sum_{n_{1}, \ldots, n_{p-1}} \tilde{a}_{n_{1}} \cdots \tilde{a}_{n_{p-1}} \tilde{a}_{k-\left(n_{1}+\ldots+n_{p-1}\right)} & \subseteq\left(\sum_{n_{1}<0} \frac{A}{\left(-n_{1}\right)^{s}} \alpha^{p-2} A^{p-1}+A \alpha^{p-2} A^{p-1}\right. \\
& \left.\quad+\sum_{n_{1}>0} \frac{A}{n_{1}^{s}} \alpha^{p-2} A^{p-1}\right)[-1,1] \\
\subseteq & \alpha^{p-2} A^{p}\left[2\left(1+\int_{1}^{\infty} t^{-s} d t\right)+1\right][-1,1] \\
= & \alpha^{p-2} A^{p}\left[\frac{3 s-2}{s-1}\right][-1,1] \\
\subset & \subset \alpha^{p-1} A^{p}[-1,1]
\end{aligned}
$$

The following inequality is needed for the case $k>0$.

$$
\sum_{n=1}^{k-1} \frac{1}{n^{s}} \frac{1}{(k-n)^{s}} \leq \frac{3.5 \cdot 2^{s}}{k^{s}}
$$

First note that we may assume $k>2$ since the sum is empty when $k=1$ and the inequality holds for the single term when $k=2$.

For $s=2$,

$$
\begin{aligned}
\sum_{n=1}^{k-1} \frac{1}{n^{2}} \frac{1}{(k-n)^{2}} & \leq \frac{2}{(k-1)^{2}}+\int_{1}^{k-1} \frac{1}{x^{2}(k-x)^{2}} d x \\
& \leq \frac{2}{(k-1)^{2}}+\frac{4}{k^{3}} \ln |k-1|+\frac{2(k-2)}{k^{2}(k-1)} \\
& \leq \frac{2}{(k-1)^{2}}+\frac{6}{k^{2}} \\
& <\frac{8}{k^{2}}+\frac{6}{k^{2}} \\
& =\frac{3.5 \cdot 2^{2}}{k^{2}}
\end{aligned}
$$

Here, $\frac{2}{(k-1)^{2}}<\frac{8}{k^{2}}$ since $k>2$.

Now assume that

$$
\sum_{n=1}^{k-1} \frac{1}{n^{s-1}(k-n)^{s-1}} \leq \frac{3.5 \cdot 2^{s-1}}{k^{s-1}}
$$

or, equivalently,

$$
\sum_{n=1}^{k-1} \frac{k^{s-1}}{n^{s-1}(k-n)^{s-1}} \leq 3.5 \cdot 2^{s-1}
$$


Then

$$
\begin{aligned}
\sum_{n=1}^{k-1} \frac{k^{s}}{n^{s}(k-n)^{s}} & =\sum_{n=1}^{k-1}\left(\frac{k}{n(k-n)}\right) \frac{k^{s-1}}{n^{s-1}(k-n)^{s-1}} \\
& \leq \frac{k}{k-1} \sum_{n=1}^{k-1} \frac{k^{s-1}}{n^{s-1}(k-n)^{s-1}} \\
& \leq 2 \cdot 3.5 \cdot 2^{s-1} \\
& =3.5 \cdot 2^{s}
\end{aligned}
$$

Therefore,

$$
\sum_{n=1}^{k-1} \frac{1}{n^{s}} \frac{1}{(k-n)^{s}} \leq \frac{3.5 \cdot 2^{s}}{k^{s}}
$$

for all $s \geq 2$.

Now, for $k>0$,

$$
\begin{aligned}
\sum_{n_{1}, \ldots, n_{p-1}} \tilde{a}_{n_{1}} \cdots \tilde{a}_{n_{p-1}} \tilde{a}_{k-\left(n_{1}+\ldots+n_{p-1}\right)} & \subseteq \sum_{n_{1}} \tilde{a}_{n_{1}} \mid \frac{\alpha^{p-2} A^{p-1}}{\left|k-n_{1}\right|^{s}}[-1,1] \\
& \subseteq\left(\sum_{n_{1}<0} \frac{A}{\left(-n_{1}\right)^{s}} \frac{\alpha^{p-2} A^{p-1}}{\left(k-n_{1}\right)^{s}}+\frac{A \alpha^{p-2} A^{p-1}}{k^{s}}\right. \\
& \left.+\sum_{n_{1}=1}^{k-1} \frac{A}{n_{1}^{s}} \frac{\alpha^{p-2} A^{p-1}}{\left(k-n_{1}\right)^{s}}+\sum_{n_{1}>k} \frac{A}{n_{1}^{s}} \frac{\alpha^{p-2} A^{p-1}}{\left(n_{1}-k\right)^{s}}\right)[-1,1] \\
& \subseteq \alpha^{p-2} A^{p}\left[\frac{2}{k^{s}}\left(1+\int_{1}^{\infty} t^{-s} d t\right)\right. \\
& \left.+\sum_{n_{1}=1}^{k-1} \frac{1}{n_{1}^{s}} \frac{1}{\left(k-n_{1}\right)^{s}}\right][-1,1] \\
\subseteq & \alpha^{p-2} A^{p}\left[\frac{2}{k^{s}}\left(1+\frac{1}{s-1}\right)+\frac{3.5 \cdot 2^{s}}{k^{s}}\right][-1,1] \\
\subseteq & \frac{\alpha^{p-2} A^{p}}{k^{s}}\left[\frac{2}{s-1}+2+3.5 \cdot 2^{s}\right][-1,1] \\
& =\frac{\alpha^{p-1} A^{p}}{k^{s}}[-1,1]
\end{aligned}
$$

The case $k<0$ may be reduced to the previous case via change of indices.

口

We may extend this argument to the maps corresponding to $c a^{p}$ provided that the expansion of the coefficient function also exhibits polynomial decay.

COROLLARY 5.9. If there exists a constant $C$ such that $c_{n} \in \tilde{c}_{n}:=\frac{C}{|n|^{s}}[-1,1]$ for all $n$, then 


$$
\sum_{n_{0}, n_{1}, \ldots, n_{p-1} \in \mathbf{Z}} \tilde{c}_{n_{0}} \tilde{a}_{n_{1}} \cdots \tilde{a}_{n_{p-1}} \tilde{a}_{k-\left(n_{0}+\ldots+n_{p-1}\right)} \subseteq\left\{\begin{array}{cc}
\frac{\alpha^{p} A^{p} C}{|k|^{s}}[-1,1] & k \neq 0 \\
\alpha^{p} A^{p} C[-1,1] & k=0
\end{array}\right.
$$

We now refine the error bounds using the explicit interval bounds $\tilde{a}_{n}$ for $n<\bar{M}$.

Lemma 5.10. For $0 \leq k<\bar{M}$,

$$
\begin{aligned}
\sum_{n_{1}, \ldots, n_{p-1} \in \mathbb{Z}} \tilde{a}_{n_{1}} \cdots \tilde{a}_{n_{p-1}} \tilde{a}_{k-\left(n_{1}+\ldots+n_{p-1}\right)} & \\
& \subseteq \sum_{\left|n_{1}\right|, \ldots,\left|n_{p-1}\right| \leq \bar{M}} \tilde{a}_{n_{1}} \cdots \tilde{a}_{n_{p-1}} \tilde{a}_{k-\left(n_{1}+\ldots+n_{p-1}\right)} \\
& \quad+\frac{(p-1) \alpha^{p-2} A^{p-1} A_{s}}{\bar{M}^{s-1}(s-1)}\left[\frac{1}{(\bar{M}-k)^{s}}+\frac{1}{(\bar{M}+k)^{s}}\right][-1,1]
\end{aligned}
$$

for any $\bar{M}>0$.

Proof.

$$
\begin{aligned}
\sum_{n_{1}, \ldots, n_{p-1} \in \mathbb{Z}} \tilde{a}_{n_{1}} \cdots \tilde{a}_{n_{p-1}} \tilde{a}_{k-\left(n_{1}+\ldots+n_{p-1}\right)} & \\
& \subseteq \sum_{\left|n_{1}\right|, \ldots,\left|n_{p-1}\right| \leq \bar{M}} \tilde{a}_{n_{1}} \cdots \tilde{a}_{n_{p-1}} \tilde{a}_{k-\left(n_{1}+\ldots+n_{p-1}\right)} \\
& \quad+\sum_{i=1}^{p-1} \max _{a_{n} \in \tilde{a}_{n}}\left\{\sum_{n_{j} \in \mathbb{Z},\left|n_{i}\right|>\bar{M}}\left|a_{n_{1}}\right| \ldots\left|a_{n_{p-1}}\right|\left|a_{k-\left(n_{1}+\cdots+n_{p-1}\right)}\right|\right\}[-1,1]
\end{aligned}
$$

Note that

$$
\sum_{n_{j} \in \mathbb{Z},\left|n_{i}\right|>\bar{M}}\left|a_{n_{1}}\right| \cdots\left|a_{n_{p-1}}\right|\left|a_{k-\left(n_{1}+\ldots+n_{p-1}\right)}\right|=\sum_{n_{j} \in \mathbb{Z},\left|n_{1}\right|>\bar{M}}\left|a_{n_{1}}\right| \cdots\left|a_{n_{p-1}}\right|\left|a_{k-\left(n_{1}+\ldots+n_{p-1}\right)}\right|
$$

for $i, \ldots, p-1$.

By Lemma 5.8,

$$
\begin{aligned}
\sum_{n_{j} \in \mathbb{Z},\left|n_{1}\right|>\bar{M}}\left|a_{n_{1}}\right| & \cdots\left|a_{n_{p-1}}\right|\left|a_{k-\left(n_{1}+\ldots+n_{p-1}\right)}\right| \\
\leq & \sum_{n_{1}>\bar{M}} \frac{A_{s}}{n_{1}^{s}} \frac{\alpha^{p-2} A^{p-1}}{\left(n_{1}-k\right)^{s}} \\
& \quad+\sum_{n_{1}<-\bar{M}} \frac{A_{s}}{\left(-n_{1}\right)^{s}} \frac{\alpha^{p-2} A^{p-1}}{\left(k-n_{1}\right)^{s}} \\
= & \alpha^{p-2} A^{p-1} A_{s} \sum_{n_{1}>\bar{M}} \frac{1}{n_{1}^{s}\left(n_{1}-k\right)^{s}}
\end{aligned}
$$




$$
\begin{aligned}
& \quad+\alpha^{p-2} A^{p-1} A_{s} \sum_{n_{1}>\bar{M}} \frac{1}{n_{1}^{s}\left(k+n_{1}\right)^{s}} \\
& \leq \frac{\alpha^{p-2} A^{p-1} A_{s}}{(\bar{M}-k)^{s}} \int_{\bar{M}}^{\infty} x^{-s} d x \\
& \quad+\frac{\alpha^{p-2} A^{p-1} A_{s}}{(\bar{M}+k)^{s}} \int_{\bar{M}}^{\infty} x^{-s} d x \\
& =\frac{\alpha^{p-2} A^{p-1} A_{s}}{\bar{M}^{s-1}(s-1)}\left[\frac{1}{(\bar{M}-k)^{s}}+\frac{1}{(\bar{M}+k)^{s}}\right]
\end{aligned}
$$

Therefore,

$$
\begin{aligned}
\sum_{n_{1}, \ldots, n_{p-1} \in \mathbb{Z}} \tilde{a}_{n_{1}} \cdots & \tilde{a}_{n_{p-1}} \tilde{a}_{k-\left(n_{1}+\ldots+n_{p-1}\right)} \\
& \subseteq \sum_{\left|n_{1}\right|, \ldots,\left|n_{p-1}\right| \leq \bar{M}} \tilde{a}_{n_{1}} \cdots \tilde{a}_{n_{p-1}} \tilde{a}_{k-\left(n_{1}+\ldots+n_{p-1}\right)} \\
& \quad+\frac{(p-1) \alpha^{p-2} A^{p-1} A_{s}}{\bar{M}^{s-1}(s-1)}\left[\frac{1}{(\bar{M}-k)^{s}}+\frac{1}{(\bar{M}+k)^{s}}\right][-1,1]
\end{aligned}
$$

口

Corollary 5.11. For $0 \leq k<\bar{M}$,

$$
\begin{aligned}
\sum_{n_{0}, \ldots, n_{p-1} \in \mathbb{Z}} \tilde{c}_{n_{0}}^{p} \tilde{a}_{n_{1}} \cdots \tilde{a}_{n_{p-1}} \tilde{a}_{k-\left(n_{0}+\ldots+n_{p-1}\right)} & \\
\subseteq & \sum_{\left|n_{0}\right|, \ldots,\left|n_{p-1}\right| \leq \bar{M}} \tilde{c}_{n_{0}}^{p} \tilde{a}_{n_{1}} \cdots \tilde{a}_{n_{p-1}} \tilde{a}_{k-\left(n_{0}+\ldots+n_{p-1}\right)} \\
& +\frac{\alpha^{p-1} A^{p} C}{\bar{M}^{s-1}(s-1)}\left[\frac{1}{(\bar{M}-k)^{s}}+\frac{1}{(\bar{M}+k)^{s}}\right][-1,1] \\
& +\frac{(p-1) \alpha^{p-1} A^{p-1} C A_{s}}{\bar{M}^{s-1}(s-1)}\left[\frac{1}{(\bar{M}-k)^{s}}+\frac{1}{(\bar{M}+k)^{s}}\right][-1,1]
\end{aligned}
$$

for any $\bar{M}>0$.

Since the sum in these last estimates is finite, we may decide on a case-by-case basis which of these terms contain only variables $\left(a_{k}\right.$ with $\left.0 \leq k<m\right)$. These terms are contained in $f^{(m)}$ and should not be included when computing bounds for $f^{(m+)}$. We may also compute a better bound of the remaining terms in the sum by using explicit interval bounds for $a_{n}, n<\bar{M}$ instead of the extended asymptotic bounds.

Corollary 5.12. For $0 \leq k<m$, the error in the $k$ th coordinate map from the neglected higher modes,

$$
\begin{aligned}
{\left[f^{(m+)}(Z)\right]_{k} \subset } & \sum_{\left|n_{0}\right|, \ldots,\left|n_{p-1}\right| \leq \bar{M}} \overline{c_{n_{0}}^{p} a_{n_{1}} \cdots a_{n_{p-1}} a_{k-\left(n_{0}+\ldots+n_{p-1}\right)}} \\
+ & \left(A+(p-1) A_{s}\right) \frac{(\alpha A)^{p-1} C}{\bar{M}^{s-1}(s-1)}\left[\frac{1}{(\bar{M}-k)^{s}}+\frac{1}{(\bar{M}+k)^{s}}\right][-1,1]
\end{aligned}
$$


where $\overline{c_{n_{0}} a_{n_{1}} \cdots a_{n_{p-1}} a_{k-\left(n_{0}+\ldots+n_{p-1}\right)}}$ is 0 if all of the indices have absolute value less than $m$ and is the interval bound $\tilde{c}_{n_{0}}^{p} \tilde{a}_{n_{1}} \cdots \tilde{a}_{n_{p-1}} \tilde{a}_{k-\left(n_{0}+\ldots+n_{p-1}\right)}$ otherwise.

The computations in this section may be used to calculate error bounds (Corollary 5.12 ) to be used in the construction of the multivalued map and to update explicit interval bounds (Corrollary 5.11). In the following lemma, we use Corollary 5.9 to update the bounds $\tilde{a}_{k}, k>M$.

LEMmA 5.13. Suppose the nonlinearity is $g(a)=\sum_{p=0}^{d} c_{p} a^{p}$ with the expansions of the coefficient functions satisfying the decay rules $c_{n}^{p} \in \tilde{c}_{n}^{p}:=\frac{C_{p}}{|n|^{s}}[-1,1]$ for some constants $C_{p}$. Then for $k \geq M$, we may set new bounds $\tilde{a}_{k}$ to be $\frac{A_{s+b}}{k^{(s+b)}}[-1,1]$ where

$$
A_{s+b}=B\left(C_{0}+C_{1} A \alpha+\cdots+C_{d} A^{d} \alpha^{d}\right)
$$

Proof.

For a fixed $k \geq M$, we have bounds for the projection onto the $k$ th mode of the image of $Z$ under the full map using the bounds for $\left|b_{k}\right|$ and the bounds for the sum given by Corollary 5.9 .

$$
\begin{aligned}
{\left[F_{m}(Z)\right]_{k} } & =b_{k} \sum_{p=0}^{d} \sum_{n_{0}, \ldots n_{p-1}} \tilde{c}_{n_{0}}^{p} \tilde{a}_{n_{1}} \cdots \tilde{a}_{k-\left(n_{0}+\ldots+n_{p-1}\right)} \\
& \subseteq \frac{B}{k^{b}} \frac{1}{k^{s}}\left(C_{0}+\alpha A C_{1}+\cdots+\alpha^{d} A^{d} C_{d}\right)[-1,1] \\
& =\frac{A_{s+b}}{k^{(s+b)}}[-1,1]
\end{aligned}
$$

where $A_{s+b}$ is as given in the statement of the lemma. We preserve isolation by setting the new bounds to be the bounds on the image.

6. Example computations. In this section we apply the previously described methodology for the analysis of the Kot-Schaffer map. We perform rigorous computations in order to prove the existence of (and compute an approximation to) the following three types of invariant sets for $\Phi$ :

1. A heteroclinic orbit connecting a neighborhood of a fixed point to a neighborhood of a period two orbit (6.1);

2. An invariant set with chaotic dynamics, i.e. with positive entropy (6.2);

3. A second complicated invariant set which is described in terms of chaotic symbolic dynamics (6.3).

The computations have been performed using the GAIO [3] and CHomP [17] packages. There are scripts available that will perform the following procedures.

6.1. Connecting orbits. In this section we show the existence of a heteroclinic orbit of $\Phi$ connecting a (small) neighborhood of a fixed point to a (small) neighborhood of a period two point and compute an approximation of this orbit to an accuracy on the order of $10^{-12}$. The parameter values for the equivalent countable system (1.4) are as follows:

$$
\mu=3.5, \quad b_{k}=2^{-k}, \quad c_{0}=0.8, \quad c_{1}=-0.2 \quad \text { and } \quad c_{k}=0 \text { for } k>1 .
$$

In order to initialize the apriori bounds $a_{k}^{ \pm}$we run a simulation of $f^{(L)}: \mathbb{R}^{L} \rightarrow \mathbb{R}^{L}$ for $L=50$ with the initial condition $A=\left\{\left(2^{-k}\right)_{k=0}^{49}\right\}, K_{0}=10$ and $K_{1}=10000$. 

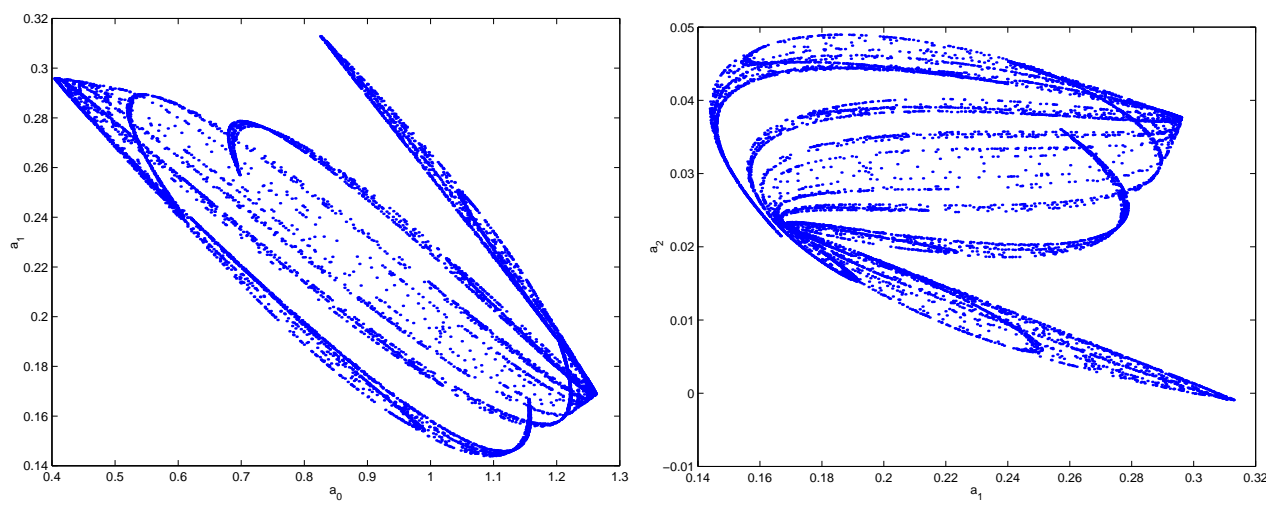

FIG. 6.1. Projections of the set $A_{K}$.

Figure 6.1 shows two projections of the resulting set $A_{K}$. We choose $M=50$ and an exponential estimate for $k \geq M$ with $A_{s}=1$ and $s=2$ as well as the initial bounds as stated in Table 6.1.

\begin{tabular}{|c|c|c|}
\hline$k$ & $a_{k}^{-}$ & $a_{k}^{+}$ \\
\hline 0 & 0.2 & 1.5 \\
1 & 0.05 & 0.5 \\
2 & -0.001 & 0.1 \\
$2<k<M$ & $-2^{-k}$ & $2^{-k}$ \\
\hline \multicolumn{2}{|c|}{ TABLE 6.1} \\
Initial guess for error bounds $a_{k}^{ \pm}$
\end{tabular}

The next step is to choose the (initial) projection dimension $m$. To this end we compute bounds for the errors $\varepsilon_{k}^{(m+)}, k=0, \ldots, m-1$, for various $m$. Based on these values (cf. Table 6.2) we decide to start off with $m=5$. Again, this is a preliminary choice and at this point in the procedure we cannot guarantee that they will be sufficient later when trying to compute isolating neighborhoods.

\begin{tabular}{|c|c|c|c|c|c|}
\hline$m$ & 2 & 3 & 4 & 5 & 6 \\
\hline & & & & 0.014583 & 0.0036458 \\
& & 0.18083 & 0.058333 & 0.011849 & 0.0029622 \\
$\varepsilon_{k}^{(m+)}$ & 0.32139 & 0.13052 & 0.040833 & 0.010208 & 0.0029622 \\
& 0.27164 & 0.19013 & 0.032630 & 0.008158 & 0.0025521 \\
& & & & 0.011883 & 0.0020394 \\
& & & & 0.0029708 \\
\hline
\end{tabular}

Errors induced by neglecting the higher order modes for different projection dimensions.

We are now able to compute guesses for invariant sets of $\mathcal{F}^{(5)}$. We do this by running Algorithm 3 with selection criterion (4.1), yielding an approximate covering of the maximal invariant set in the chosen region $W^{(5)}=\prod_{k=0}^{4}\left[a_{k}^{-}, a_{k}^{+}\right]$. In course of the algorithm the coordinate direction $j$ in which the boxes are subdivided varies cyclically with the number of subdivisions $k$, i.e. $j=k \bmod 5$. For computing the multivalued 
map $\mathcal{F}^{(5)}$ on the collections $\mathcal{B}_{k}$ we employ the heuristic method of mapping test points, here we use a set of 100 points per box distributed randomly according to a uniform distribution. Figure 6.2 shows two projections of the resulting box collection in $\mathbb{R}^{5}$ after 35 steps of the algorithm.
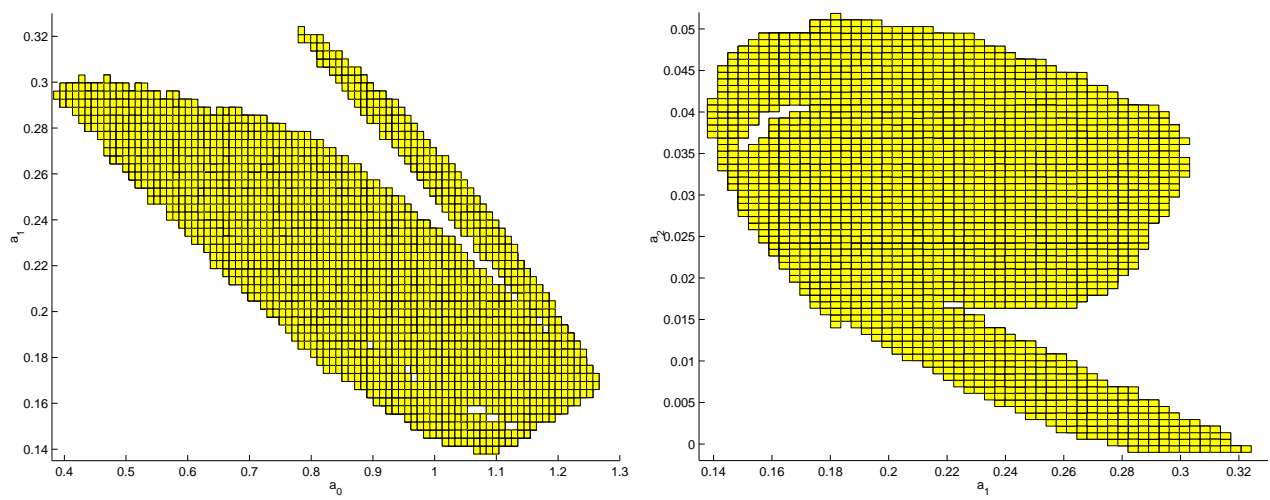

FIG. 6.2. Projections of the (approximate) box covering $\mathcal{B}_{35}$ of the maximal invariant set of $F^{(5)}$ in $W^{(5)}$.

Before we proceed with extracting the desired dynamical objects from the collection $\mathcal{B}_{35}$ we update the apriori bounds $a_{k}^{ \pm}, k=5, \ldots, 49$, (see Section 4.3.2) in order to reduce the error $\varepsilon^{(m+)}$. The resulting $\varepsilon^{(m+)}$ using the updated bounds is smaller than $(0.05,0.1,0.2,0.6,1.25)^{T} \cdot 10^{-4}$. Note that this error (rather than the initial error as shown in Table 6.2) determines whether we will be able to do a useful computation later on. It essentially sets a lower bound to the size of the boxes used to cover the objects of interest. In our case $\varepsilon^{(m+)}$ is roughly (at least) four times smaller than the radius of the boxes in $\mathcal{B}_{35}$.

Recall that our aim is to compute an orbit connecting a fixed point to a period two point of $\Phi$. Guesses for isolating neighborhoods of the periodic points of $f^{(5)}$ are easily obtained by considering the periodic points of $\mathcal{F}^{(5)}: \mathcal{B}_{35} \rightrightarrows \mathcal{B}_{35}$ as laid out in Section 4.1. Figure 6.3 shows the guess $\tilde{\mathcal{I}}_{1}$ for the fixed points and the guess $\tilde{\mathcal{I}}_{2}$ for the period two points. Finally we obtain a guess $\tilde{\mathcal{I}}_{c}$ for a connecting orbit by computing the shortest path from the box in $\tilde{\mathcal{I}}_{1}$ to some box in $\tilde{\mathcal{I}}_{2}$ (cf. Figure $6.3(\mathrm{c}$ ), see again Section 4.1). Using the collection $\tilde{\mathcal{I}}_{c}$ as input we now intend to use Algorithm 2 to compute an isolating neighborhood for the multivalued map $F^{(5)}$. To this end we need to deal with a (true) enclosure of $F^{(5)}$ (in contrast to the heuristic one we used so far in order to obtain our initial guesses). Bounds on the errors $\varepsilon^{(m+)}$ are computed using the results of Section 5.2 and updated using the procedures discussed in Section 4.3.2.

By running Algorithm 2 with input $\tilde{\mathcal{I}}_{c}$ now we obtain a combinatorial isolating neighborhood $\mathcal{I}$ for $\mathcal{F}^{(5)}$ (cf. Figure 6.4). The union $|\mathcal{I}|$ of these boxes is an isolating neighborhood for $F^{(5)}$ (cf. [22]). Using (1.13) we finally compute a corresponding index pair $\left(\left|\mathcal{N}_{1}\right|,\left|\mathcal{N}_{0}\right|\right)$. Figure 6.5 shows two different views of these sets, where the exit set $\left|\mathcal{N}_{0}\right|$ corresponds to the red boxes. The resulting relative homology groups of the pair $N=\left(\left|\mathcal{N}_{1}\right|,\left|\mathcal{N}_{0}\right|\right)$ are

$$
H_{*}\left(\left|\mathcal{N}_{1}\right|,\left|\mathcal{N}_{0}\right|\right) \cong\left(0, \mathbb{Z}^{7}, 0,0, \ldots\right) .
$$

Obviously, the associated homology map $f_{N *}^{(5)}: H_{*}\left(\left|\mathcal{N}_{1}\right|,\left|\mathcal{N}_{0}\right|\right) \rightarrow H_{*}\left(\left|\mathcal{N}_{1}\right|,\left|\mathcal{N}_{0}\right|\right)$ induced by $f_{N}^{(5)}$ is trivial everywhere but level one. For an appropriate choice of basis 


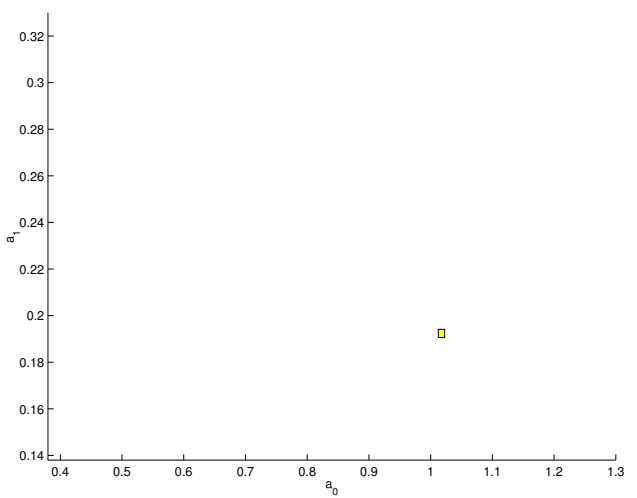

(a)

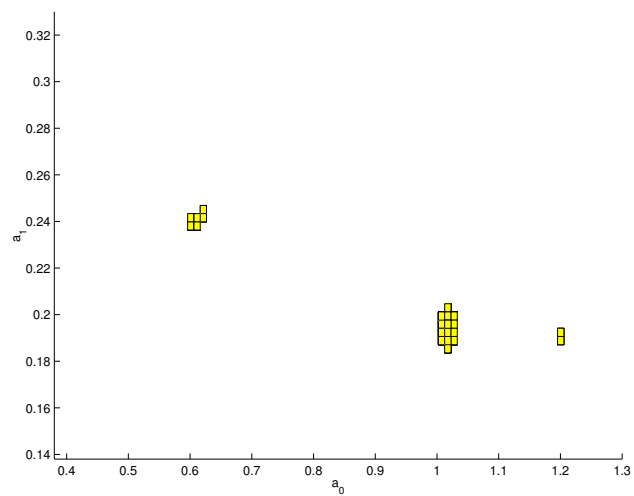

(b)

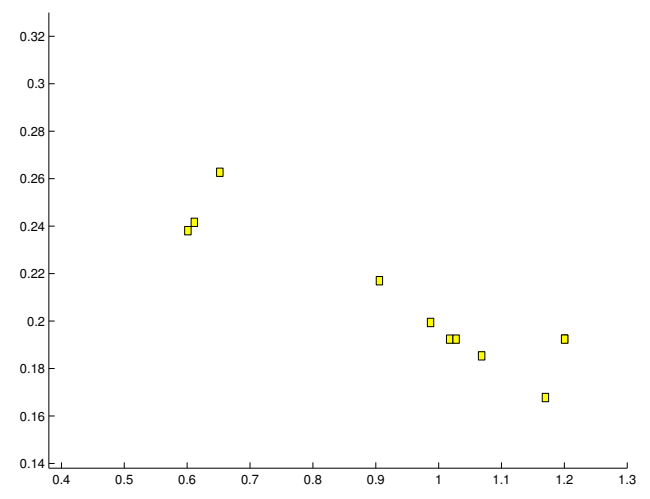

(c)

FIG. 6.3. Guesses for isolating neighborhoods of (a) the fixed points, (b) the period two points (note that the fixed points are covered, too) and (c) a connecting orbit of $f^{(5)}$ (projections onto the first two coordinates).
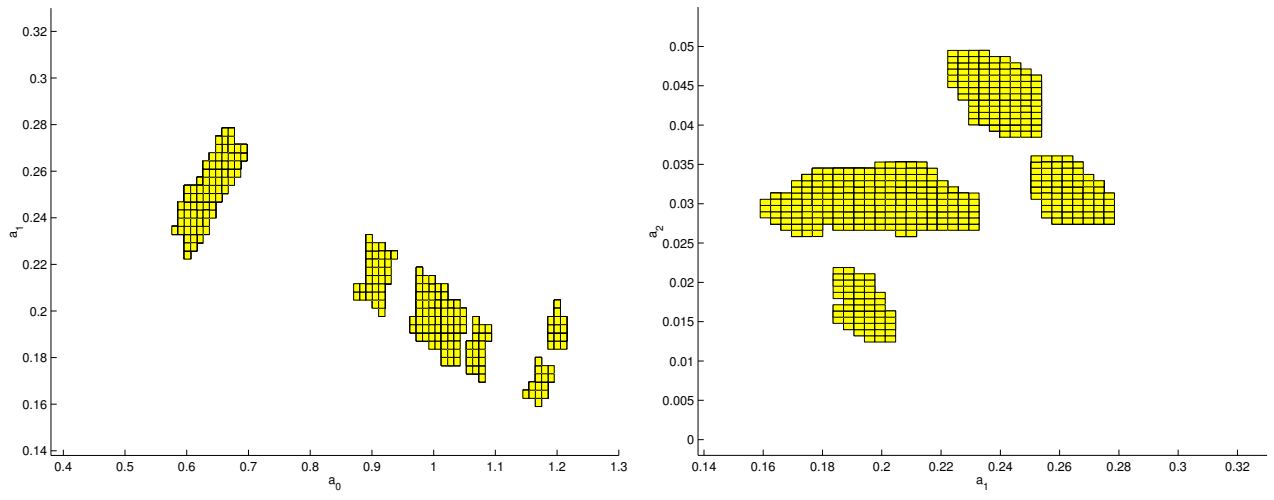

FIG. 6.4. Combinatorial isolating neighborhood for $\mathcal{F}^{(5)}$ (projections onto the first and the second two coordinates, respectively). 

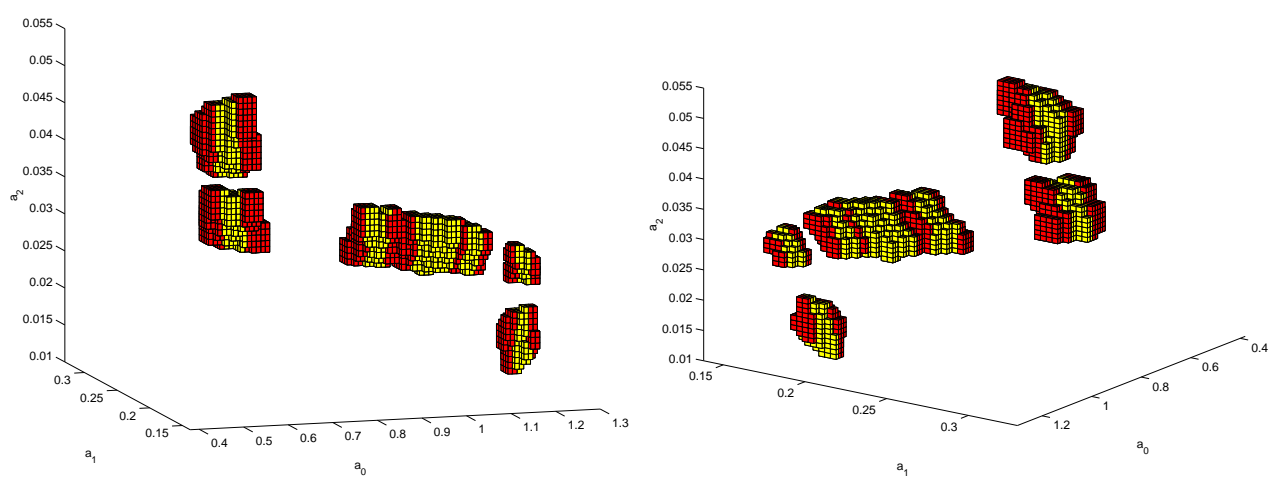

FIG. 6.5. Index pair for the multivalued map $F^{(5)}$ (projections onto the first three coordinates). The red boxes correspond to the exit set $\left|\mathcal{N}_{0}\right|$.

$f_{N, 1}^{(5)}$ can be expressed as the following matrix,

$$
f_{N, 1}^{(5)}:=\left[\begin{array}{ccccccc}
-1 & 0 & 0 & 0 & 0 & 0 & 0 \\
1 & 0 & 0 & 0 & 0 & 0 & 0 \\
0 & 1 & 0 & 0 & 0 & 0 & 0 \\
0 & 0 & 1 & 0 & 0 & 0 & 0 \\
0 & 0 & 0 & 1 & 0 & 0 & 0 \\
0 & 0 & 0 & 0 & 1 & 0 & 1 \\
0 & 0 & 0 & 0 & 0 & -1 & 0
\end{array}\right]
$$

An important observation is that each generator of $H_{1}\left(\left|\mathcal{N}_{1}\right|,\left|\mathcal{N}_{0}\right|\right)$ lies in a distinct connected component of $\left|\mathcal{N}_{1}\right| \backslash\left|\mathcal{N}_{0}\right|$. Using the same notation as in Section 2.2 we label these components by $B_{1}, \ldots, B_{7}$. The multivalued map $F^{(5)}$ provides us with the following information concerning the dynamics between the boxes:

1. $F^{(5)}\left(B_{1}\right) \cap B_{j} \neq \emptyset$ only if $j=1,2$.

2. For $i=2, \ldots, 6, F^{(5)}\left(B_{i}\right) \cap B_{j} \neq \emptyset$ only if $j=i+1$.

3. $F^{(5)}\left(B_{7}\right) \cap B_{j} \neq \emptyset$ only if $j=6$.

In particular, we can consider the following index maps, obtained from $f_{N, 1}^{(5)}$ by projection onto the corresponding connected components.

$$
f_{B_{1}, 1}^{(5)}:=\left[\begin{array}{ccccccc}
-1 & 0 & 0 & 0 & 0 & 0 & 0 \\
1 & 0 & 0 & 0 & 0 & 0 & 0 \\
0 & 0 & 0 & 0 & 0 & 0 & 0 \\
0 & 0 & 0 & 0 & 0 & 0 & 0 \\
0 & 0 & 0 & 0 & 0 & 0 & 0 \\
0 & 0 & 0 & 0 & 0 & 0 & 0 \\
0 & 0 & 0 & 0 & 0 & 0 & 0
\end{array}\right] \quad f_{B_{6} \cup B_{7}, 1}^{(5)}:=\left[\begin{array}{ccccccc}
0 & 0 & 0 & 0 & 0 & 0 & 0 \\
0 & 0 & 0 & 0 & 0 & 0 & 0 \\
0 & 0 & 0 & 0 & 0 & 0 & 0 \\
0 & 0 & 0 & 0 & 0 & 0 & 0 \\
0 & 0 & 0 & 0 & 0 & 0 & 0 \\
0 & 0 & 0 & 0 & 0 & 0 & 1 \\
0 & 0 & 0 & 0 & 0 & -1 & 0
\end{array}\right] .
$$

Observe that $\operatorname{tr} f_{B_{1}}^{(5)}=-1$ and $\operatorname{tr}\left(f_{B_{6} \cup B_{7}}^{(5)}\right)^{2}=-2$.

There are several conclusions that can be drawn from this information. First, $\operatorname{Inv}\left(B_{1}, f^{(5)}\right) \neq \emptyset$ and $\operatorname{Inv}\left(B_{6} \cup B_{7}, f^{(5)}\right) \neq \emptyset$. In fact, by $(2.5)$ we know that $\operatorname{Inv}\left(B_{1}, f^{(5)}\right)$ contains a fixed point and $\operatorname{Inv}\left(B_{6} \cup B_{7}, f^{(5)}\right)$ contains a periodic point. The second and third pieces of information concerning the multivalued map $F^{(5)}$ indicate that this periodic orbit has minimal period 2 . 
Finally, $f_{N, 1}^{(5)}$ is not shift equivalent to $f_{B_{1}, 1}^{(5)} \oplus f_{B_{6} \cup B_{7}, 1}^{(5)}$, thus the Conley indices of $\operatorname{Inv}\left(I, f^{(5)}\right)$ and $\operatorname{Inv}\left(B_{1} \cup B_{6} \cup B_{7}, f^{(5)}\right)$ are different. This in turn implies that $\operatorname{Inv}\left(I, f^{(5)}\right) \neq \operatorname{Inv}\left(B_{1} \cup B_{6} \cup B_{7}, f^{(5)}\right)$. Again, returning to the information concerning the multivalued map $F^{(5)}$ we can conclude that $\operatorname{Inv}\left(I, f^{(5)}\right)$ must contain an orbit whose omega limit set is contained in $B_{6} \cup B_{7}$ and whose alpha limit set is contained in $B_{1}$.

Since these results concerning the existence of orbits follow from the algebra of the Conley index, after checking condition (2.4) we can immediately carry over this existence result to the infinite dimensional system (1.4) and thus (1.1).

However, we aim for a more precise localization of the detected objects and continue with tightening the computed covering as described in Section 4.3. We end up with a collection $\mathcal{I}^{(11)}$ of 24078 boxes in $\mathbb{R}^{11}$ and - employing Corollaries 2.4 and 2.5 - the following theorem.

THEOREM 6.1. For the parameter values (6.1) the map $\Phi$ possesses the following orbits all of which lie in the set

$$
\left|\mathcal{I}^{(11)}\right| \times \prod_{k=11}^{49}\left[a_{k}^{-}, a_{k}^{+}\right] \times \prod_{k=50}^{\infty} \frac{1}{2^{k}}[-1,1],
$$

where the $a_{k}^{ \pm}$are the final bounds:

1. A fixed point $p$ with the property that

$$
\left\|p-p^{*}\right\|_{L^{2}}<3 \cdot 10^{-12}, \quad\left\|p-p^{*}\right\|_{C^{0}}<3.6 \cdot 10^{-12},
$$

where the Fourier coefficients of $p^{*}$ (given in Table 6.3) are determined by $B_{1}$.

2. A periodic orbit $q=\left\{q_{1}, q_{2}\right\}$ with the property that

$$
\left\|q_{i}-q_{i}^{*}\right\|_{L^{2}}<3.3 \cdot 10^{-12}, \quad\left\|q_{i}-q_{i}^{*}\right\|_{C^{0}}<4.3 \cdot 10^{-12}, \quad i=1,2,
$$

where the Fourier coefficients of $q_{1}^{*}$ and $q_{2}^{*}$ (given in Table 6.4) are determined by $B_{6}$ and $B_{7}$, respectively.

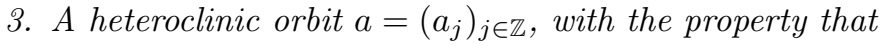

$$
\delta(\alpha(a), p)<3.5 \cdot 10^{-12}, \quad \delta(\omega(a), q)<3.8 \cdot 10^{-12},
$$

where $\delta(\cdot, \cdot)$ is the distance in the Hausdorff metric on compact sets.

6.2. Positive entropy. As a second example we now reveal the presence of complicated dynamics for the parameter values chosen in the previous example.

Using the methods employed in the previous example we first construct an isolating neighborhood which contains a heteroclinic cycle between a fixed point and a period two point of $\Phi$. To this end we compute guesses $\tilde{\mathcal{I}}_{c_{12}} \subset \mathcal{B}_{36}$ and $\tilde{\mathcal{I}}_{c_{21}} \subset \mathcal{B}_{36}$ for orbits connecting the fixed point to a period two point of $\mathcal{F}^{(5)}$ and vice versa as described in the previous example. Then we use Algorithm 2 with input $\tilde{\mathcal{I}}_{c_{12}} \cup \tilde{\mathcal{I}}_{c_{21}}$ to construct an isolating neighborhood $\mathcal{I}^{(5)}$ for $\mathcal{F}^{(5)}$. A corresponding index pair $\left(\mathcal{N}_{1}\right.$, $\mathcal{N}_{0}$ ) is shown in Figure 6.6.

The relative homology of the index pair $N=\left(\left|\mathcal{N}_{1}\right|,\left|\mathcal{N}_{0}\right|\right)$ for $f^{(5)}$ is

$$
H_{*}\left(\left|\mathcal{N}_{1}\right|,\left|\mathcal{N}_{0}\right|\right) \cong\left(0, \mathbb{Z}^{14}, 0,0, \ldots\right)
$$




\begin{tabular}{|l|l|}
\hline$k$ & $\left(p^{*}\right)_{k}$ \\
\hline 0 & 1.01701222469896 \\
1 & 0.194337336695483 \\
2 & 0.030518985313998 \\
3 & 0.00388416812157288 \\
4 & 0.000406689870061427 \\
5 & $3.59686642677538 \cdot 10^{-5}$ \\
6 & $2.75312512992254 \cdot 10^{-6}$ \\
7 & $1.85783274541004 \cdot 10^{-7}$ \\
8 & $1.12063486092261 \cdot 10^{-8}$ \\
9 & $6.10776632026745 \cdot 10^{-10}$ \\
10 & $3.03735627584813 \cdot 10^{-11}$ \\
$\geq 11$ & 0 \\
\hline
\end{tabular}

Fourier coefficients of $p^{*}$.

\begin{tabular}{|l|l|l|}
\hline$k$ & $\left(q_{1}^{*}\right)_{k}$ & $\left(q_{2}^{*}\right)_{k}$ \\
\hline 0 & 0.612404314978377 & 1.20068110795964 \\
1 & 0.240548407216622 & 0.191505924758062 \\
2 & 0.0427803169880592 & 0.0186218996298865 \\
3 & 0.00371012084664812 & 0.00293452057215404 \\
4 & 0.000286872331997756 & 0.000493085588431086 \\
5 & $2.85759422150114 \cdot 10^{-5}$ & $4.83837782923445 \cdot 10^{-5}$ \\
6 & $2.46114548652796 \cdot 10^{-6}$ & $3.16438225821576 \cdot 10^{-6}$ \\
7 & $1.61009990418761 \cdot 10^{-7}$ & $1.78892095960431 \cdot 10^{-7}$ \\
8 & $9.62062994427963 \cdot 10^{-9}$ & $1.00726290466515 \cdot 10^{-8}$ \\
9 & $5.95120614532175 \cdot 10^{-10}$ & $5.28784988682338 \cdot 10^{-10}$ \\
10 & $3.43628779469884 \cdot 10^{-11}$ & $2.46555443216212 \cdot 10^{-11}$ \\
$\geq 11$ & 0 & 0 \\
\hline
\end{tabular}

Fourier coefficients of $q_{1}^{*}$ and $q_{2}^{*}$.

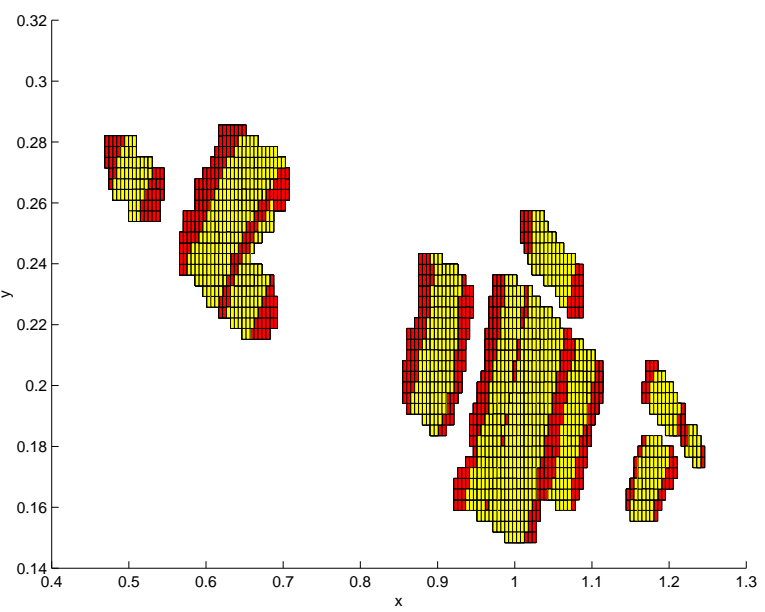

FIG. 6.6. Index pair for $\mathcal{F}^{(5)}$ (projection onto the first two coordinates). The yellow boxes cover the invariant set, the red boxes constitute the exit set.

37 
and the map $f_{N *}^{(5)}: H_{*}\left(\left|\mathcal{N}_{1}\right|,\left|\mathcal{N}_{0}\right|\right) \rightarrow H_{*}\left(\left|\mathcal{N}_{1}\right|,\left|\mathcal{N}_{0}\right|\right)$ induced by $f^{(5)}$ in homology is trivial at all levels but level one on which $f_{N *}^{(5)}$ acts on the 14 ordered generators as follows

$$
f_{N, 1}^{(5)}=\left[\begin{array}{llllllllllllll}
0 & 0 & 0 & 0 & 0 & 0 & 0 & 0 & 0 & 0 & 1 & 0 & 1 & 0 \\
0 & 0 & 0 & 0 & 0 & 0 & 0 & 0 & 0 & 0 & 0 & 1 & 0 & 0 \\
0 & 0 & 0 & 0 & 0 & 0 & 1 & 1 & 0 & 0 & 0 & 1 & 0 & 0 \\
0 & 0 & 0 & 1 & 0 & 0 & 0 & 0 & 0 & 0 & 0 & 0 & 0 & 0 \\
0 & 0 & 0 & 0 & 1 & 0 & 0 & 0 & 0 & 1 & 0 & 0 & 0 & 0 \\
0 & 0 & 0 & 0 & 1 & 0 & 0 & 0 & 0 & 1 & 0 & 0 & 0 & 0 \\
0 & 0 & 0 & 0 & 0 & 0 & 0 & 0 & 0 & 0 & 0 & 0 & 0 & 1 \\
0 & 0 & 0 & 0 & 0 & 1 & 0 & 0 & 0 & 0 & 0 & 0 & 0 & 0 \\
0 & 0 & 0 & 0 & 0 & 0 & 0 & 0 & 1 & 0 & 0 & 0 & 0 & 0 \\
0 & 0 & 0 & 0 & 0 & 0 & 0 & 0 & 1 & 0 & 0 & 0 & 0 & 0 \\
1 & 0 & 0 & 0 & 0 & 0 & 0 & 0 & 0 & 0 & 0 & 0 & 0 & 0 \\
0 & 0 & 1 & 0 & 0 & 0 & 0 & 0 & 0 & 0 & 0 & 0 & 0 & 0 \\
0 & 1 & 0 & 0 & 0 & 0 & 0 & 0 & 0 & 0 & 0 & 0 & 0 & 0 \\
0 & 1 & 0 & 0 & 0 & 0 & 0 & 0 & 0 & 0 & 0 & 0 & 0 & 0
\end{array}\right] .
$$

The spectral radius of $f_{N, 1}^{(5)}$ is bigger than 1.2, therefore - according to [2] and using Corollary 2.5 - we have the following theorem.

THEOREM 6.2. For the values given in (6.1), there exists an invariant set contained in

$$
\left|\mathcal{I}^{(5)}\right| \times \prod_{k=6}^{49}\left[a_{k}^{-}, a_{k}^{+}\right] \times \prod_{k=50}^{\infty} \frac{1}{2^{k}}[-1,1], \quad j \in \mathbb{Z},
$$

(where $\left[a_{k}^{-}, a_{k}^{+}\right]$are the final bounds), on which $\Phi$ exhibits positive entropy.

6.3. A 2d-unstable horseshoe. As a last example we prove the existence of and localize another invariant set on which $\Phi$ exhibits complicated dynamics. In contrast to the previous computation this time we aim for the dimension of its unstable manifold to be two.

We are considering the map (1.4) with the following values for its parameters:

$$
\begin{aligned}
& \mu=3.8, \quad b_{0}=1, \quad b_{1}=0.99, \quad b_{2}=0.1, \quad b_{3}=0.025, \quad b_{k}=2^{-k}, k \geq 3, \\
& c_{0}=0.8, \quad c_{1}=-0.2 \quad \text { and } \quad c_{k}=0 \text { for } k>1 .
\end{aligned}
$$

Again we start by running a simulation of $f^{(L)}: \mathbb{R}^{L} \rightarrow \mathbb{R}^{L}$ for $L=100$ with the initial point $A=\left\{\left(10^{-4}, 0, \ldots, 0\right)\right\}, K_{0}=100$ and $K_{1}=50000$. Figure 6.7 shows two projections of the resulting set $A_{K}$. We choose $m=6, M=50$ and an exponential estimate for $k \geq M$ with $A_{s}=1$ and $s=2$ as well as the initial bounds as indicated in Table 6.5 .

Again, in order to compute guesses for invariant sets we first compute a covering of the maximal invariant set of $F^{(6)}$ by running 44 steps of Algorithm 3 with selection criterion (4.1). We employ the heuristic method of mapping 200 randomly distributed (according to a uniform distribution) test points per box in order to compute $\mathcal{F}^{(6)}$. The resulting collection consists of 1130128 boxes. As in the previous examples we now update the bounds. The updated bounds lead to an error $\varepsilon^{(m+)}$ which is smaller than $\left(2 \cdot 10^{-7}, 5 \cdot 10^{-7}, 2 \cdot 10^{-7}, 2 \cdot 10^{-6}, 7 \cdot 10^{-6}, 2 \cdot 10^{-5}\right)^{T}$. Similar to the previous example we compute guesses $\tilde{\mathcal{I}}_{c_{12}} \subset \mathcal{B}_{44}$ and $\tilde{\mathcal{I}}_{c_{21}} \subset \mathcal{B}_{44}$ for orbits connecting the fixed 

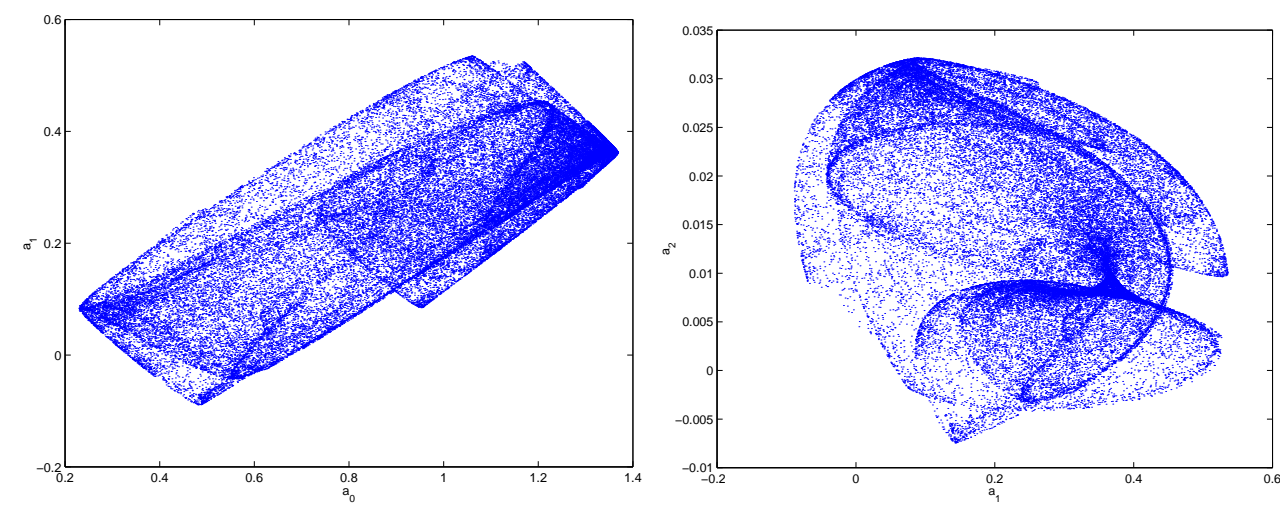

FIG. 6.7. Projections of the set $A_{K}$.

\begin{tabular}{|c|c|c|}
\hline$k$ & $a_{k}^{-}$ & $a_{k}^{+}$ \\
\hline 0 & 0.1601 & 1.7781 \\
1 & -0.06131 & 0.70231 \\
2 & -0.0036184 & 0.04173 \\
3 & -0.00032171 & 0.033337 \\
$3<k<M$ & $-2^{-k}$ & $2^{-k}$ \\
\hline \multicolumn{3}{|c|}{ Tnitial guess for error bounds $a_{k}^{ \pm}}$.
\end{tabular}

point to a period two point of $F^{(5)}$ and vice versa. Again we use Algorithm 2 with input $\tilde{\mathcal{I}}_{c_{12}} \cup \tilde{\mathcal{I}}_{c_{21}}$ to construct an isolating neighborhood $\mathcal{I}^{(6)}$ for $\mathcal{F}^{(6)}$. The relative homology of the corresponding index pair $N=\left(\left|\mathcal{N}_{1}\right|,\left|\mathcal{N}_{0}\right|\right)$ for $f^{(6)}$ is

$$
H_{*}\left(\left|\mathcal{N}_{1}\right|,\left|\mathcal{N}_{0}\right|\right) \cong\left(0, \mathbb{Z}, \mathbb{Z}^{18}, 0,0, \ldots\right) .
$$

The neighborhood $\left|\mathcal{N}_{1}\right|$ consists of 11 connected components, $A, B, \ldots, K$. We label the 18 generators of $H_{2}\left(\left|\mathcal{N}_{1}\right|,\left|\mathcal{N}_{0}\right|\right)$ according to their location in these components as shown in Figure 6.8. The combinatorial map $\mathcal{F}^{(6)}$ on the boxes induces a map on the components $A, B, \ldots, K$. This map is shown in Figure 6.8(a), together with the homology map on $H_{2}\left(\left|\mathcal{N}_{1}\right|,\left|\mathcal{N}_{0}\right|\right)$ (Fig. 6.8(b)).

We now use the index information to prove that there exists a set contained in $\left|\mathcal{N}_{1}\right| \times \Pi_{k \geq 6}\left[a_{k}^{-}, a_{k}^{+}\right]$on which the full map $\Phi$ exhibits symbolic dynamics as depicted by the transition graph $T_{1}$ shown in Figure 6.8(a).

Let $\Sigma=\left\{\left(Z_{i}\right)_{i \in \mathbb{Z}} \mid Z_{i} \in\{A, B, \ldots, K\}\right\}$ and let $\sigma: \Sigma \rightarrow \Sigma$ be the shift map. Consider the subset, $\Sigma_{*}=\left\{\left(Z_{i}\right)_{i} \in \Sigma \mid\left(Z_{i}, Z_{i-1}\right)\right.$ is an edge in $\left.T_{1}\right\}$. As in Section 2, define $\rho:\left|\mathcal{N}_{1}\right| \rightarrow \Sigma_{*}$ by $\rho(x)=\left(Z_{i}\right)_{i \in \mathbb{Z}}$, where $Z_{i}$ is the connected component of $\left|\mathcal{N}_{1}\right|$ containing $\left(f^{(6)}\right)^{i}(x), i \in \mathbb{Z}$. A result of [20] allows us to decompose the index information into maps on the connected components of $\left|\mathcal{N}_{1}\right|$. Let $f_{Z, 2}^{(6)}, Z \in$ $\{A, \ldots, K\}$, be the index map obtained from $f_{N, 2}^{(6)}$ by projecting onto the connected component $Z$. According to Szymczak in [23], for every $n$ there exists at least one trajectory under $f^{(6)}$ through $Z_{1}, \ldots, Z_{n}$ provided that the composition $f_{Z_{1}, 2}^{(6)} \circ \ldots \circ$ $f_{Z_{n}, 2}^{(6)}$ is not nilpotent. In the limit, any infinite sequence of symbols in $\Sigma_{*}$ corresponds to at least one trajectory under $f^{(6)}$ through the given components. Furthermore if 


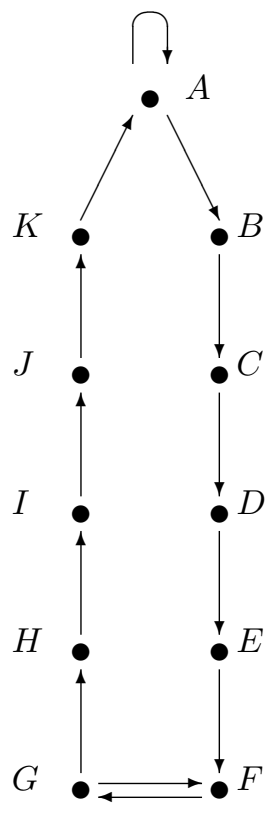

(a)

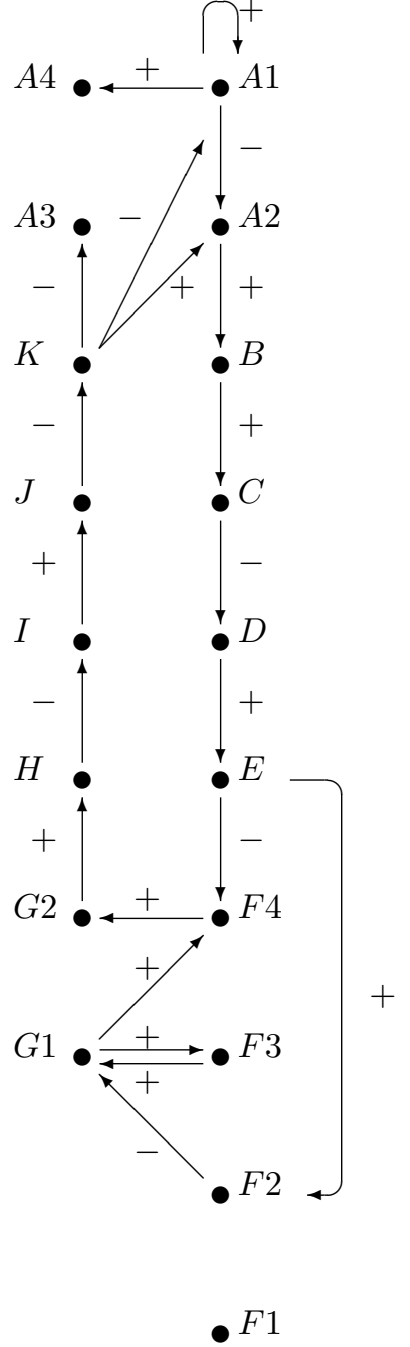

(b)

FIG. 6.8. (a) The graph $T_{1}$. It indicates the existence of 11 connected components of the isolating neighborhood. The arrows indicate how the connected component are related by the map $F^{(6)}$. (b) The graph $T_{2}$. This graph indicates the relative homology classes within each connected component and the edges indicate how the generators of these homology classes are mapped one into the other. The \pm signs indicate whether the matrix entry for $F_{2}^{(6)}: H_{2}\left(N_{1}, N_{0}\right) \rightarrow H_{2}\left(N_{1}, N_{0}\right)$ is \pm 1 .

the Lefschetz number of $f_{Z_{1} *}^{(6)} \circ \ldots \circ f_{Z_{n} *}^{(6)}$ is nonzero, then a periodic orbit under $f^{(6)}$ through components $Z_{1}, \ldots, Z_{n}$ exists (see also Equation 2.5). Since trace $f_{N, i}^{(6)}=0$ for $i \neq 2$ (we have $f_{N, 1}^{(6)}=0$ ), the Lefschetz number is nonzero provided that the trace of the composition of restricted index maps for $H_{2}\left(\left|\mathcal{N}_{1}\right|,\left|\mathcal{N}_{0}\right|\right)$ is nonzero. This can be shown for the composition of restricted index maps for any periodic sequence given by the transition graph in Figure 6.8. By extension, $\rho$ maps onto $\Sigma_{*}$, the closure of periodic orbits given in the transition graph $T_{1}$ shown in Figure 6.8(a).

By construction, $\rho$ is a semi-conjugacy between $f^{(6)}$ on the (nonempty) invariant 
set contained in $\left|\mathcal{N}_{1}\right| \backslash\left|\mathcal{N}_{0}\right|$ and the shift map $\sigma$ on $\Sigma_{*}$. After checking that the lifting condition (2.4) is satisfied, we have the following theorem.

THEOREM 6.3. For the parameter values (6.2) there is an invariant set contained in $\left|\mathcal{N}_{1}\right| \times \Pi_{k \geq 6}\left[a_{k}^{-}, a_{k}^{+}\right]$(where $\left[a_{k}^{-}, a_{k}^{+}\right]$are the final bounds), on which $\Phi$ is semiconjugate to the subshift given by the transition graph $T_{1}$ shown in Figure 6.8(a).

Acknowledgements. The authors would like to thank Madjid Allili and Paweł Pilarczyk for providing code and assistance in doing the homology computations. KM and SD were partially supported by NSF grants DMS 0107396, DMS 9805584 and INT 9910027 . OJ was partially supported by DAAD/NSF grant D/0103717.

\section{REFERENCES}

[1] G. Arioli and P. Zgliczyński, Symbolic dynamics for the Hénon-Heiles Hamiltonian on the critical level, J. Differential Equations, 171 (2001), pp. 173-202.

[2] A. W. BAKER, Lower bounds on entropy via the Conley index with application to time series, Topology Appl., 120 (2002), pp. 333-354.

[3] M. Dellnitz, G. Froyland, And O. Junge, The algorithms behind GAIO-set oriented numerical methods for dynamical systems, in Ergodic theory, analysis, and efficient simulation of dynamical systems, Springer, Berlin, 2001, pp. 145-174, 805-807.

[4] M. Dellnitz and A. Hohmann, A subdivision algorithm for the computation of unstable manifolds and global attractors, Numer. Math., 75 (1997), pp. 293-317.

[5] M. Dellnitz, O. Junge, M. Rumpf, and R. Strzodka, The computation of an unstable invariant set inside a cylinder containing a knotted flow, in Proceedings of Equadiff '99, Berlin, 2000.

[6] E. W. Dijkstra, A note on two problems in connexion with graphs, Numer. Math., 1 (1959), pp. 269-271.

[7] M. Eidenschink, Exploring Global Dynamics: A Numerical Algorithm Based on the Conley Index Theory, PhD thesis, Georgia Institute of Technology, 1995.

[8] J. Franks and D. Richeson, Shift equivalence and the Conley index, Trans. Amer. Math. Soc., 352 (2000), pp. 3305-3322.

[9] J. K. Hale And G. Raugel, Regularity, determining modes and galerkin methods. Preprint, CDSNS, Georgia Institite of Technology, 2002.

[10] O. Junge, Rigorous discretization of subdivision techniques, in Proceedings of Equadiff '99, Berlin, 2000.

[11] T. Kaczynski, K.Mischaikow, and M. Mrozek, Computational Homology, In preparation, 2003.

[12] T. Kaczynski and M. Mrozek, Conley index for discrete multivalued dynamical systems, Topology and its applications, 65 (1995), pp. 83-96.

[13] O. KNüPpel, BIAS - Basic Interval Arithmetic Subroutines, Technische Universität HamburgHarburg, 1993.

[14] —, PROFIL - Programmer's Runtime Optimized Fast Interval Library, Technische Universität Hamburg-Harburg, 1993.

[15] M. Kot and W. M. Schaffer, Discrete-time growth-dispersal models, Math. Biosci., 80 (1986), pp. 109-136.

[16] K. Mischaikow and M. Mrozek, Conley index, in Handbook of Dynamical Systems, Elsevier, Berlin, 2002, pp. 393-460.

[17] K. Mischaikow, M. Mrozek, and P. Pilarczyk, Graph approach to the computation of the homology of continuous maps. In preparation.

[18] K. Mischaikow, M. Mrozek, and A. Szymczak, Chaos in the Lorenz equations: a computer assisted proof. III. Classical parameter values, J. Differential Equations, 169 (2001), pp. 1756. Special issue in celebration of Jack K. Hale's 70th birthday, Part 3 (Atlanta, GA/Lisbon, 1998).

[19] M. MrozeK, Shape index and other indices of Conley type for local maps on locally compact Hausdorff spaces, Fund. Math., 145 (1994), pp. 15-37.

[20] A. Szymczak, The Conley index for decompositions of isolated invariant sets, Fund. Math., 148 (1995), pp. 71-90.

[21] - The Conley index and symbolic dynamics, Topology, 35 (1996), pp. 287-299.

$[22]$ - A combinatorial procedure for finding isolating neighbourhoods and index pairs, Proc. Roy. Soc. Edinburgh Sect. A, 127 (1997), pp. 1075-1088. 
[23] - Index Pairs: from Dynamics to Combinatorics and Back, PhD thesis, Georgia Institute of Technology, 1999.

[24] W. Tucker, A rigorous ODE solver and Smale's 14th problem, Found. Comput. Math., 2 (2002), pp. 53-117.

[25] J. Zemke, b4m, A free interval arithmetic toolbox for Matlab based on BIAS, Technische Universität Hamburg-Harburg, http://www.ti3.tu-harburg.de/zemke/b4m.

[26] P. ZGLiczyŃSKi AND K. Mischaikow, Rigorous numerics for partial differential equations: the Kuramoto-Sivashinsky equation, Found. Comp. Math., 1 (2001), pp. 255-288. 